\title{
Reference Tables for 40 Percent Iridium-60 Percent Rhodium Versus Iridium Thermocouples*
}

\author{
G. F. Blackburn and F. R. Caldwell
}

(June 6, 1961)

\begin{abstract}
In a program to establish reference tables for several alloys of iridium and rhodium against iridium, the work has been completed on 40 percent iridium-60 percent rhodium. Tables have been prepared giving emfs for temperatures in degrees Fahrenheit from $32{ }^{\circ} \mathrm{F}$ to $3,800{ }^{\circ} \mathrm{F}$ and in degrees Celsius (centigrade) from $0{ }^{\circ} \mathrm{C}$ to $2,100{ }^{\circ} \mathrm{C}$, and temperatures in these units with emf in millivolts as the argument.

The emf is 11.6 millivolts at $3,800{ }^{\circ} \mathrm{F}$. The thermoelectric power averages 3.1 microvolts per ${ }^{\circ} \mathrm{F}$, and is within 0.1 microvolt of this value between $500{ }^{\circ} \mathrm{F}$ and $3,000{ }^{\circ} \mathrm{F}$. It increases somewhat at temperatures above $3,000{ }^{\circ} \mathrm{F}$.

Measurements at temperatures $32{ }^{\circ} \mathrm{F}$ to $2,500{ }^{\circ} \mathrm{F}$ were made in a platinum-wound furnace of conventional design, using a Pt-10 percent $\mathrm{Rh}$ versus $\mathrm{Pt}$ thermocouple to measure temperature. From 1,950 ${ }^{\circ} \mathrm{F}$ to $3,800{ }^{\circ} \mathrm{F}$ (thus overlapping the upper end of the lower range), the furnace used was an iridium block heated by electric induction. Blackbody conditions prevailed in the cavity in which the test thermocouple was inserted, and enabled measurement of the temperature with an optical pyrometer.

Good agreement between the data obtained in the overlapping parts of the platinum and optical pyrometer ranges $\left(1,950^{\circ} \mathrm{F}\right.$ to $\left.2,500^{\circ} \mathrm{F}\right)$ established confidence in the temperature measurements with the optical pyrometer above $2,500{ }^{\circ} \mathrm{F}$.

In both furnaces, helium surrounded the thermocouple junctions. The gas was dried by passing it through a trap immersed in liquid nitrogen.

Thoria and beryllia were used as thermal and electrical insulation in the high temperature range. While the electrical resistivity of these materials becomes relatively low at high temperatures, it did not become so low as to cause appreciable error in emf measurements. More serious was the introduction into the measuring circuit of a spurious emf, associated with the strong rf field and believed to be due to a rectifying property possessed by the insulators at high temperature. It was present in varying degrees, sometimes not at all. Its magnitude was determined by observing the (instantaneous) change in emf when the rf power was turned off, and the appropriate correction was made in the thermocouple emf measured with the power on.

The accuracy of the measurements is largely that associated with the measurement of temperature using an optical pyrometer. For the present work the maximum uncertainties in the range $2,500{ }^{\circ} \mathrm{F}$ to $3,800^{\circ} \mathrm{F}$ are believed to range from about $8{ }^{\circ} \mathrm{F}$ to $12{ }^{\circ} \mathrm{F}$.
\end{abstract}

\section{Introduction}

One of the earliest investigations of thermocouples of alloys of iridium and rhodium versus iridium was that made by Feussner [1]. ${ }^{1}$ His studies led him to suggest the alloy 40 percent iridium-60 percent rhodium for use with iridium. The increasing need in recent years for thermocouples capable of measuring temperatures higher than those at which platinum-rhodium versus platinum thermocouples can be used has led to further work on this combination [2], along with improvements in the metallurgy of these metals. In turn, the gradually increasing use of this and other iridium-rhodium allovs as thermoelements has given rise to the need for reference tables of the type available for platinum-rhodium versus platinum and other thermocouples [3].

*This paper was presented at the Fourth Symposium on Temperature, Its Measurement and Control in Science and Industry, Columbus, Ohio, March 27-31, 1961, sponsored by the American Institute of Physics, the Instrument Society of America, and the National Bureau of Standards. The proceedings will be published in book form by the Reinhold Publishing Corporation, New York, N.Y.

1 Figures in brackets indicate the literature references at the end of this paper.
The work reported here evolved from a project at the National Bureau of Standards originally sponsored by the United States Air Force, to examine thermocouples of alloys of iridium with 10 percent to 90 percent rhodium versus iridium. The tables (Appendix, I-A to IV-A) give the temperature-electromotive force values for 40 percent iridium-60 percent rhodium versus iridium, the first of the series to be completed.

\section{Apparatus}

Calibration up to $2,500{ }^{\circ} \mathrm{F}$ is accomplished in a high-temperature porcelain tube furnace having a bifilar platinum heating coil. The temperature is measured with a platinum-10 percent rhodium versus platinum thermocouple, calibrated prior to each run.

A blackbody is used to calibrate at higher temperatures. It is an iridium cylinder $17 / 16$ in. in diameter and $45 / 16$ in. long, with cavities as shown in figure 1. 


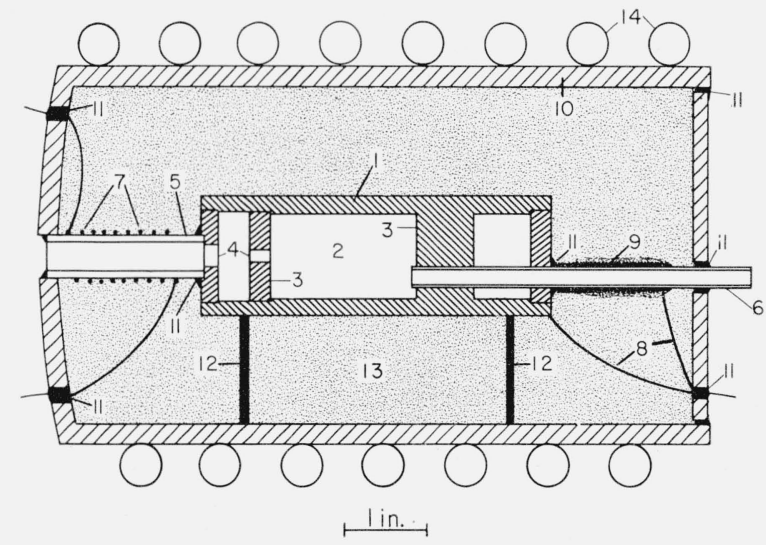

Figure 1. Cross section of induction furnace.

1, blackbody; 2 , central cavity; 3 , partitions; 4 , sighting holes; 5 , sighting tube 6 , thermocouple entrance tube; 7 , heater winding; 8 , ground wires; 9 , iridium shield; 10 , porcelain can; 11 , thoria cement; 12 , thoria supports; 13 , thoria powder; 14, copper tubing.

Alined holes are provided in one end and the adjacent partition for inserting the thermocouple in to the central cavity, and in the other end for sighting with the optical pyrometer which is used to measure the temperature. The area sighted upon is on the cavity wall opposite the $1 / 8$-in. diameter hole in the partition at this end.

It is assumed that, owing to the design and method of heating the blackbody, the only departure from uniform temperature over the wall of the central cavity is that due to loss of heat by radiation through holes. This loss as calculated by a formula due to Gouffé [4] is about 0.2 percent, which is so small that it is unnecessary to correct observed temperatures on this account.

The blackbody is heated by electric induction with a $20 \mathrm{kw}$ radiofrequency generator. A coil of iridium wire surrounds the sight tube and is connected externally to an rf ammeter and rheostat. The heat produced by rf current in the coil reduces the loss of heat from that end of the blackbody. A similar arrangement could be used at the thermocouple end; however, in an effort to provide shielding for the thermocouple wires and supporting tube, iridium wire was wound in a tight coil that formed a shield over the protection tube. Being heated by induction, this shield also serves to reduce the loss of heat by conduction along the thermocouple wires and supporting tube.

Preliminary experiments showed that it is necessary to ground the blackbody; this is done through an iridium wire welded to it. The shield just described is also grounded, but the thermocouple is not.

Thoria powder insulates the blackbody, which is supported centrally in a porcelain can 4 in. in diameter and 8 in. long by semicircular thoria disks. A coil of $3 / 8-i n$. copper tubing surrounding the porcelain can conducts the rf current and carries cooling water.

This assembly is placed with its axis horizontal on Pyrex glass supports in a box made of Transite and Plexiglas and having close fitting but not air tight joints. In use, helium flows into the box continuously at a rate judged sufficient to keep air out. The helium is cooled to the temperature of liquid nitrogen before it enters the furnace enclosure in order to remove water vapor and any other condensable impurities which may be present.

The emf is measured with Leeds and Northrop potentiometers, Models K2 and K3. An L. and N. No. 8622 optical pyrometer is used to measure temperature. Instead of using the temperature scale of the instrument, the temperature is obtained from calibration tables giving the temperature as a function of the current through the pyrometer lamp. A 2-ohm rheostat connected in series with the slide wire of the pyrometer increases the precision with which the lamp current can be adjusted to match the filament brightness with that of the blackbody. The lamp current is determined by measuring the voltage across a 1-ohm precision resistor in series with the lamp.

\section{Thermocouples}

The thermocouple wire was made by Sigmund Cohn Corporation. The first lot (A), obtained in 1955 , was 0.035 in. in diameter. The second (B), obtained in 1959, consisted of 0.020 - and 0.035-in. diam. wire. A third lot (D) of alloy wire only, acquired in 1960, consisted of wire sizes 0.020 in. and $0.030 \mathrm{in}$.

The data used in compiling the tables were obtained from a total of eight thermocouples - two from lot $A$, two from each wire size in lot $B$, and one from each size wire in lot D. The alloy wire of lot D was joined to iridium wire of lot B. The 0.030 -in. alloy joined to 0.035 -in. iridium wire formed one thermocouple, and the two 0.020 -in. wires the other.

Each thermocouple was identified by a number indicating the lot of wire from which it was made, the percentage of rhodium in the alloy leg (" 6 ," signifying. 60 percent rhodium, is the only composition considered in this report), the wire diameter, and its order in a series of similar thermocouples. Thus A635a was thermocouple (a) from wire of lot A, 60 percent rhodium, $0.035 \mathrm{in}$. in diameter. Thermocouple (b) from the same coils of wire was A635b. The other six thermocouples were B620a, B620b, B635a, B635b, D620B, and D630B. In the last two, "B" signifies that the iridium leg was made from wire of lot $\mathrm{B}$. In the remainder of this report, a particular thermocouple will be referred to by its number.

\section{Experimental Procedure}

In preparing a thermocouple the wires were annealed by heating them electrically in air. The manufacturer recommended annealing for about $1 \mathrm{~min}$. at approximately $200{ }^{\circ} \mathrm{C}\left(360^{\circ} \mathrm{F}\right)$ below the melting. point. The temperature to be reached as observed with an optical pyrometer was estimated, taking the emissivity as 0.3 , to be $3,460{ }^{\circ} \mathrm{F}$ for the iridium and $3,180^{\circ} \mathrm{F}$ for the alloy wire [5]. In approaching these temperatures it was necessary to increase the current gradually, so that it was not unusual for several minutes to elapse while the temperature was 
being raised and pyrometric observations made. Measurement errors were comparatively large under these conditions, due in part to "smoke" that frequently appeared around the wires when they were heated to high temperature in air. For this and other reasons the actual temperature probably differred from those desired by as much as $100^{\circ} \mathrm{F}$.

The junction was welded in an oxyacetylene flame.

Each thermocouple wire joined a copper wire in a bath of melting ice. The copper wires led to a potentiometer through a selector switch.

In working with the induction furnace an undesirably large $\mathrm{rf}$ voltage was induced on the thermocouple wires. Most of this was prevented from reaching the potentiometer by inserting a 1-mh choke in each lead between the reference junction and the potentiometer, and a $1-\mu \mathrm{f}$ capacitor from the low potential end of each choke to ground.

Except for a few readings at intervals of $25^{\circ} \mathrm{F}$ on lot A thermocouples at temperatures under $300{ }^{\circ} \mathrm{F}$, the measurements were made at intervals of $50{ }^{\circ} \mathrm{F}$.

The thermocouples were run first in the $2,500{ }^{\circ} \mathrm{F}$ furnace. They were supported in high-temperature porcelain tubes and immersed about 8 in. For these runs the test and the reference thermocouples were welded to form a common junction. This made it possible to compare one thermoelement with another, and the readings regularly included measurements of the emf between the iridium and platinum, and between the iridium-rhodium and platinum elements. Each reading consisted of two measurements of each desired emf, made in such sequence that the mean values were very nearly free from error due to the slight change in temperature which unavoidably occurred while the measurements were being made.

Thermocouples A635a and A635b were each run twice, both times at ascending temperatures. The others were each run once, readings being obtained first at ascending, then descending temperatures. The means of the two readings were taken as the experimentally determined temperature-emf relationship for the thermocouple. This applies also to measurements made in the induction furnace.

For calibrating in the induction furnace a thermocouple was supported in an insulating tube of thoria or beryllia. Experiments on the effect of depth of immersion showed that, to be free of error due to conduction of heat away from the junction, the thermocouple should extend not less than $3 / 4$ in. into the central cavity. The immersion was at least twice this distance.

In making a single observation, the heating rate was adjusted so as to maintain as nearly as possible constant temperature. Then one observer adjusted the current in the pyrometer lamp until the brightness of the filament was the same as that of the blackbody. Simultaneously another observer measured the thermocouple emf. A "reading" at a given temperature was taken as the mean of two observed values of filament current and corresponding emf.

Readings were made from $1,950{ }^{\circ} \mathrm{F}$ to $3,800{ }^{\circ} \mathrm{F}$ at intervals of $50^{\circ} \mathrm{F}$, and repeated in descending order.

\section{Computations}

The observed temperatures seldom differed more than $5^{\circ} \mathrm{F}$ from the nearest integral multiple of $50^{\circ} \mathrm{F}$. For each observed temperature the emf was "normalized"- that is, it was computed for the nearest integral multiple of $50{ }^{\circ} \mathrm{F}$. The rate of change of emf with temperature $(d E / d T)$ required in normalizing was obtained either by direct calculation from observed differences or by plotting these differences and hand fitting a curve through the points.

The emf for lot A wire was taken as the mean of the normalized emfs for the two thermocouples made of that wire. Similarly, the emf for each of the two wire sizes in lot B was computed as the mean of the normalized emfs of the two thermocouples made from each of the two sizes of wires. To these emfs were added the normalized emfs obtained from lot D (one thermocouple from each of the two wire sizes). The average of these five sets of normalized emfs constituted the basic data for constructing temperature-emf tables.

These data, comprising 78 temperatures and the corresponding emfs, were processed by the Computation Section of the NBS Applied Mathematics Division using its IBM 704 Computer. It was found by trial that an equation of the fifth degree with coefficients computed to give a least-square fit to the data gave calculated emfs from which the experimental values differed by not more than $4 \mu \mathrm{v}$ at temperatures up to $2,700^{\circ} \mathrm{F}$, and not more than 10 $\mu \mathrm{v}$ at temperatures between $2,800^{\circ} \mathrm{F}$ and $3,800^{\circ} \mathrm{F}$. These deviations were considered satisfactorily small; hence, using the equation so derived, the computer calculated emfs for temperatures in integral multiples of $10^{\circ} \mathrm{F}$ from $40^{\circ} \mathrm{F}$ to $3,800{ }^{\circ} \mathrm{F}$. The machinecomputed deviations were plotted and a smooth curve fitted to them by hand. From this curve the deviations for the $10{ }^{\circ} \mathrm{F}$ points were taken, and added to the emfs computed by machine to give table IV-A.

The table of emfs for temperature in integral multiples of $10{ }^{\circ} \mathrm{C}$ (table II-A) was interpolated by hand from the Fahrenheit table.

By hand interpolation the temperatures in degrees $\mathrm{C}$ and degrees $\mathrm{F}$ were obtained for emfs in steps of 0.1 $\mathrm{mv}$ from 0 to $11.6 \mathrm{mv}$. The computer interpolated within the $0.1-\mathrm{mv}$ intervals to obtain temperatures at 0.01-mv intervals (tables I-A and III-A).

Thermal emfs for iridium versus platinum up to $2,500{ }^{\circ} \mathrm{F}$ were computed from measurements made in the course of the runs on the iridium-rhodium versus iridium thermocouples. The iridium came from three coils, viz, lot $\mathrm{A}$ and the two wire sizes of lot B. By applying corrections obtained from data on the emf of the platinum working standard versus platinum 27, the iridium versus platinum 27 values given in table $\mathrm{V}$-A were obtained.

Thermal emfs for 40 percent iridium-60 percent rhodium versus copper and for copper versus iridium are given in table VI-A. This table may be used to 
correct for emfs generated at the junctions of the thermocouple legs with copper lead wires when these junctions are not at the same temperature.

\section{Discussion}

Not surprisingly, the emfs for a given temperature differed appreciably within the three lots of wire. One way of expressing the difference is to compare the individual emfs with the average, at a given temperature. For example, at $3,000{ }^{\circ} \mathrm{F}$ the emfs were as shown in table 1 .

TABLE 1. Deviation of the thermal emf of different lots of wire from the average, at $3000^{\circ} \mathrm{F}$

\begin{tabular}{|c|c|c|c|}
\hline Lot & $\begin{array}{l}\text { Wire } \\
\text { diam }\end{array}$ & $\underset{\mathrm{E}}{\mathrm{emf}}$ & $\begin{array}{l}\text { Difference, } \\
\mathrm{E}-\mathrm{E}_{\mathrm{av}}\end{array}$ \\
\hline $\begin{array}{l}\mathrm{A} \\
\mathrm{B} \\
\mathrm{B} \\
\mathrm{D} \\
\mathrm{D}\end{array}$ & $\begin{array}{r}\text { in. } \\
0.035 \\
.020 \\
.035 \\
.020 \\
.030\end{array}$ & $\begin{array}{l}m v \\
8.830 \\
8.909 \\
8.862 \\
8.864 \\
8.842\end{array}$ & $\begin{aligned} & \mu v \\
&-31 \\
& 48 \\
& 1 \\
& 3 \\
&-19\end{aligned}$ \\
\hline A verage & & 8.861 & \\
\hline
\end{tabular}

Here the lowest emf was for lot $\mathrm{A}$ and the highest the 0.020 -in. wire of lot B. The difference is $79 \mu \mathrm{v}$, which corresponds to about $24^{\circ} \mathrm{F}$.
Similar data are plotted for the entire range of temperatures in figure 2. Differences shown there are not wholly attributable to the different sources of wire in volved, since there were naturally differences in emf between thermocouples made of wire from the same coil. In most cases these differences were only a few microvolts, but in one case, the 0.20 -in. wire of lot $\mathrm{B}$, the two thermocouples gave emfs differing by as much as $36 \mu \mathrm{v}$, corresponding to about $12^{\circ} \mathrm{F}$.

Figure 3 is a plot, for iridium wire from the three sources (lot $A$, and the two sizes in lot $B$ ) versus platinum 27, of the differences between individual values and the adjusted [6] average of the three. An emf on this graph is a measure of the contribution of the iridium in question to the analagous difference shown in figure 2 for the iridium-rhodium versus iridium thermocouple of which it is an element. For if, of two iridium wires making a common junction with an iridium-rhodium and a platinum wire, one has a higher emf to platinum than the other, its emf to the alloy wire must be lower by the same amount numerically. The reasoning can easily be extended to apply to the difference from the average (the ordinates of figs. 2 and 3 ). Thus it is evident that the lower emf of a thermocouple of lot $\mathrm{A}$ as compared with one from another lot is largely accounted for by the fact that the iridium wire of

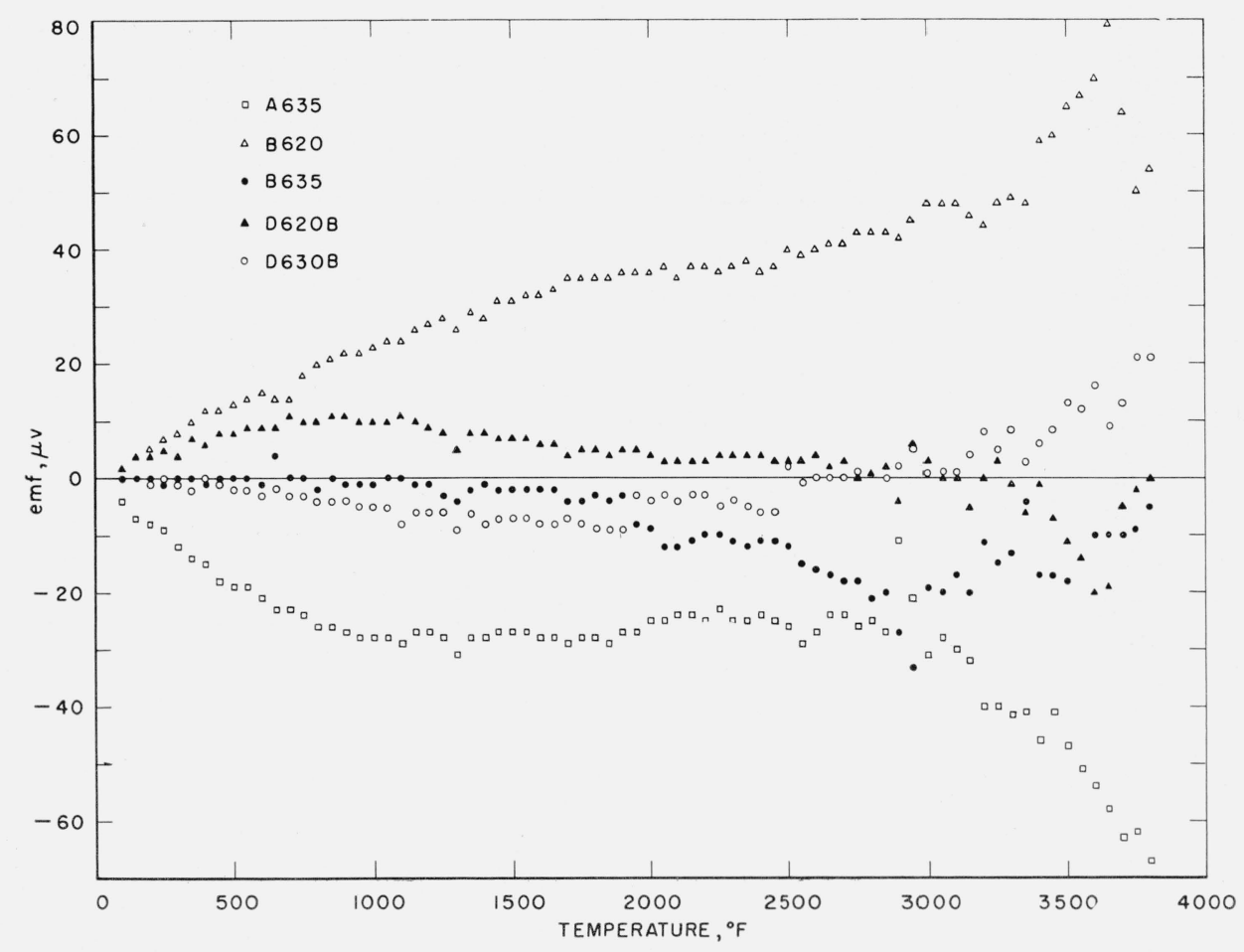

Figure 2. Difference between the emf of each thermocouple and the average emf for $40 \%$ Ir $60 \%$ Rh versus Ir thermocouples. 


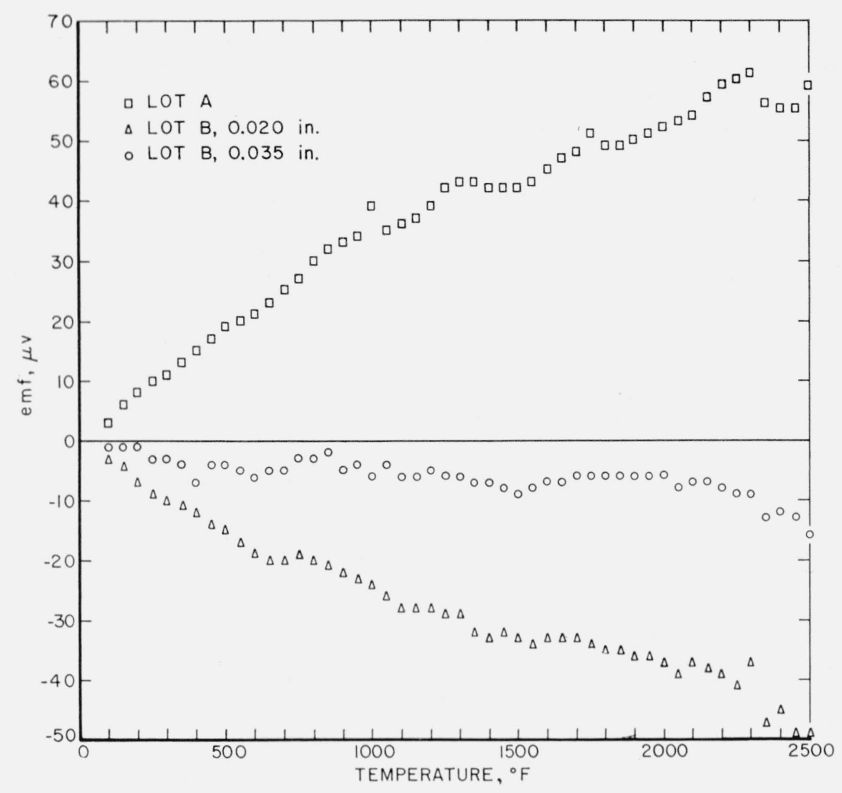

Figure 3. Difference between the emf of each lot and size of Ir wire and the average emf for Ir versus Pt 27.

this lot had a higher emf to platinum than did either of the other two coils of iridium wire. A similar situation prevails with respect to the 0.020 -in. iridium wire of lot $\mathrm{B}$. However, this wire was also used in D620B, and the differences between the emfs of figure 2 for thermocouples B620 and D620B are of course due to the differences between the two alloy wires used. These differences can be, and were, measured directly.

In the range $1,950{ }^{\circ} \mathrm{F}$ to $2,500{ }^{\circ} \mathrm{F}$, where temperatures were measured both with the platinum-10 percent rhodium thermocouple (in the platinumwound furnace) and with the optical pyrometer (in the induction furnace), the difference between emfs of the test thermocouples did not exceed $6 \mu \mathrm{v}$ in most cases. This is little if any larger than the errors of observation, and gave added assurance that the measurements were free from significantly large systematic errors. The largest difference occurred on thermocouple B635b: The emfs with the optical pyrometer averaged $21 \mu \mathrm{v}$ higher than with the platinum thermocouple. This corresponds to about $7{ }^{\circ} \mathrm{F}$, and is larger than the reading error. The data yielded no clue as to what caused the difference.

The thermoelectric power is nearly constant over a wide range of temperature, having a value of 3.0 to $3.2 \mu \mathrm{v}$ per degree from about $600^{\circ} \mathrm{F}$ to $3,200^{\circ} \mathrm{F}$. Below $600{ }^{\circ} \mathrm{F}$ it decreases to about $2 \mu \mathrm{v}$ per degree at $100{ }^{\circ} \mathrm{F}$, and above $3,200{ }^{\circ} \mathrm{F}$ it increases to about $3.6 \mu \mathrm{v}$ per degree at $3,800^{\circ} \mathrm{F}$.

The uncertainty in the temperature-emf values is partly that associated with measuring the temperature with a thermocouple and with an optical pyrometer. For the platinum-10 percent rhodium versus platinum thermocouple the uncertainty is about one degree at $2,000^{\circ} \mathrm{F}$ and 3 degrees at $2,500{ }^{\circ} \mathrm{F}[7]$. For the optical pyrometer the uncertainty was minimized by carefully calibrating the instrument in terms of lamp current and by adjusting this current precisely in the photometric matching. This procedure together with favorable blackbody conditions probably limited the uncertainty to 5 or 6 degrees at $3,800^{\circ} \mathrm{F}$.

The increased electrical conductivity of insulators at high temperature contributes to the uncertainty of the emf measurements at temperatures above about $3,200^{\circ} \mathrm{F}$. When the thoria tubes which were used at first to support the wires in the induction furnace exhibited lowered resistance they were replaced with beryllia, which has higher resistivity than thoria [8]. Even so, the resistance between thermocouple wires (as determined in a test made with the wires unjoined) decreased to a few tens of obms at $3,800{ }^{\circ} \mathrm{F}$. The magnitude of error due to insulator conduction depends on the resistance of the insulator as compared with the resistance of the thermocouple wires between points where they effectively make electrical contact with the insulator. For example, for wire resistance $0.1 \mathrm{ohm}$ between points of contact, an insulator resistance of $100 \mathrm{ohms}$ would cause the observed emf of the thermocouple to be 0.1 percent lower than the actual emf. The available data yield an estimated uncertainty of this order, which at the highest temperature would amount to about $4^{\circ} \mathrm{F}$. Since this conductivity effect is believed to have occurred in a largely fortuitous manner, no correction has been made for it.

An effect was associated with the electrical conduction in the ceramic insulation which manifested itself in the superposition of a spurious emf on the thermal emf due to the presence of the strong rf field. This was thought to result from a rectifying process. It was not always present, but when it was, readings were obtained that were larger or smaller than the thermal emf by as much as $100 \mu \mathrm{v}$. Its magnitude was ascertained by turning off the power and observing the instantaneous change in galvanometer deflection, which occurred before an appreciable change due to cooling took place. The readings were corrected by the appropriate amount. We believe that loss in precision arising from this effect was held to a few microvolts by the means adopted to correct for it.

The combined uncertainties are believed not to exceed $12{ }^{\circ} \mathrm{F}$.

The wires were discolored somewhat after a test. Although they were embrittled as a result of heating, and evidently had undergone considerable recrystallization, their thermoelectric properties were not changed appreciably. This was evidenced by good agreement of emfs measured in the low temperature range after a high temperature calibration.

The temperature-emf relationship for 40 percent iridium-60 percent rhodium versus iridium thermocouples is plotted in figure 4 for temperatures 2,000 ${ }^{\circ} \mathrm{F}$ to $3,800{ }^{\circ} \mathrm{F}$, along with the thermal emfs of several other thermocouples useful in this range, all having. 


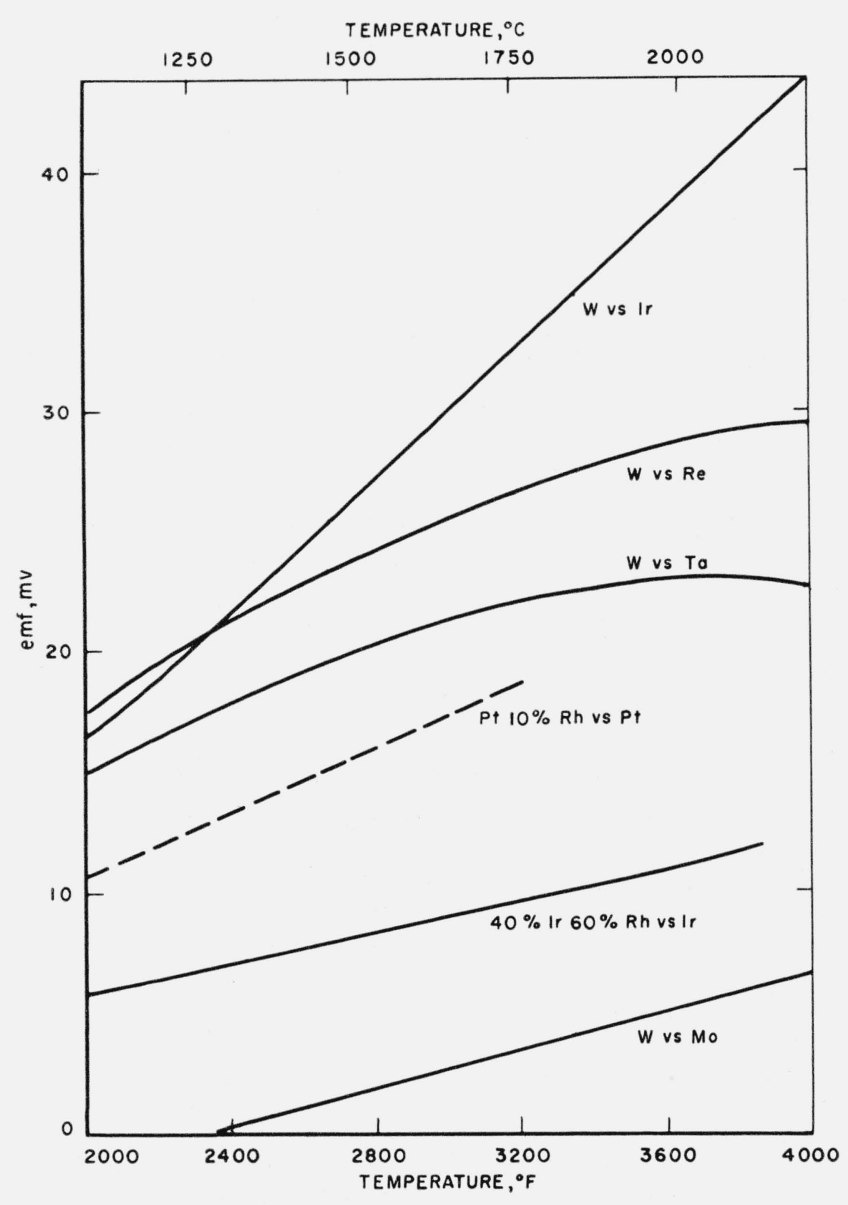

Figure 4. Thermal emf of several thermocouples in the range $2,000^{\circ} \mathrm{F}$ to $4,000^{\circ} \mathrm{F}$.

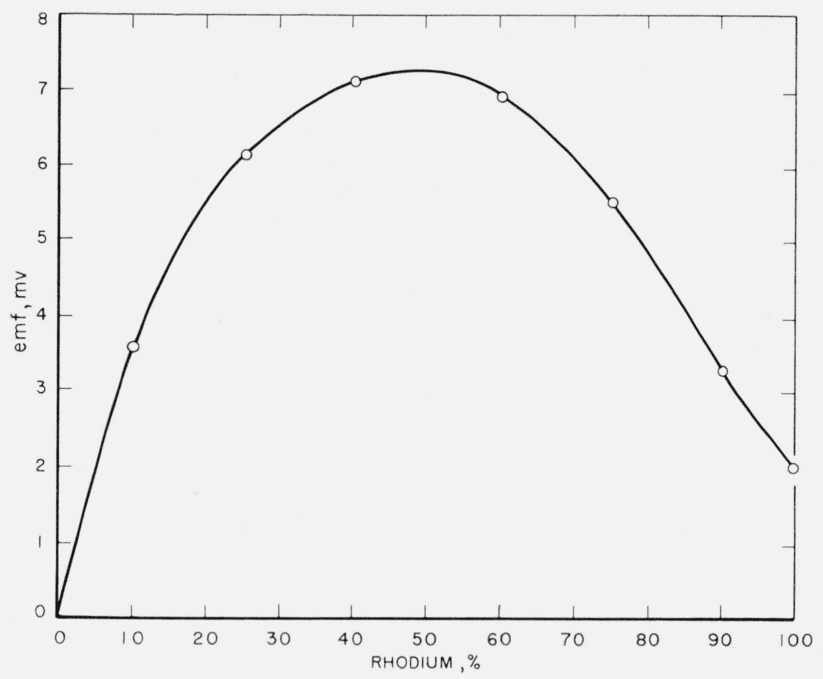

Figure 5. Change of thermal emf of Ir-Rh versus Ir thermocouples with composition of the alloy, at $2,400^{\circ} \mathrm{F}$. tungsten as an element. The data on tungsten versus iridium are from Troy and Steven [9]. For tungsten versus rhenium the data were obtained from Lachman [10], for tungsten versus molybdenum from Potter and Grant [11], and tungsten versus tantalum from Sanders [12]. The emf is also plotted for platinum-10 percent rhodium versus platinum [3].

Some data have been obtained at NBS on the effect of varying the percent rhodium in the alloy leg. They have thus far been limited to temperatures at which a platimun-rhodium versus platimun thermocouple can be used to measure the temperature. These data are plotted for one temperature, $2,400{ }^{\circ} \mathrm{F}$, in figure 5 .

The authors thank J. F. Swindells and Henry Kostkowski for permission to use the facilities of the Temperature Physics Section of the NBS Heat Division, and for their helpful interest in the work; also, to Ronald C. Gordon and D. B. Thomas for calibrating, respectively, the optical pyrometer and platinum-rhodium versus platinum thermocouple used.

They are also indebted to G. M. Galler and B. S. Prush of the Computation Section of the NBS Applied Mathematics Division for their help in arranging the data for processing by the IBM 704 Computer.

\section{References}

[1] O. Feussner, New noble-metal thermoelements for very high temperatures (in German), Elektrotech. Z. 54, 155 (1933).

[2] C. R. Droms and A. I. Dahl, Iridium vs iridium rhodium thermocouple for gas temperature measurements up to $3500{ }^{\circ} \mathrm{F}$, General Electric Company Report No. R54GL314 (Nov. 19, 1954).

[3] Henry Shenker, John I. Lauritzen, Jr., Robert J. Corruccini, and S. T. Lonberger, Reference tables for thermocouples, Natl. Bur. Standards (U.S.) Circ. $\mathbf{5 6 1}$ (1955).

[4] A. Gouffé, Opening corrections of artificial blackbodies computed on the basis of multiple internal diffusion (in French), Rev. optique $\mathbf{2 4}, 1$ (1945).

[5] Paul D. Foote, C. O. Fairchild, and T. R. Harrison, Pyrometric practice, Tech. Pap. BS 14 (1921).

[6] The averages as computed were adjusted by inspection to smooth the differences corresponding to $50{ }^{\circ} \mathrm{F}$. The change did not exceed 3 microvolts in most cases.

[7] W. F. Roeser and S. T. Lonberger, Methods of testing thermocouples and thermocouple materials, Natl. Bur. Standards (U.S.) Circ. 590 (1958).

[8] I. E. Campbell, High temperature technology (Wiley, $1956)$, p. 58

[9] W. C. Troy and Gary Steven, The tungsten iridium thermocouple for very high temperatures, Trans. Am. Soc. Metals 42, 1131 (1950).

[10] J. C. Lachman, Calibration of rhenium molybdenum and rhenium tungsten thermocouples to $4000^{\circ} \mathrm{F}$, Report APEX 365, General Electric Company, Atomic Products Division (1958).

[11] R. D. Potter and N. J. Grant, Tungsten molybdenum thermocouples, Iron Age 163, 65 (March 31, 1949).

[12] V. D. Sanders, Review of high-temperature immersion thermal sensing devices for in-flight engine control, Rev. Sci. Instr. 29, 917 (1958). 


\section{Appendix}

TABLE I-A. Forty percent iridium-60 percent rhodium versus iridium thermocouples

Electromotive force in absolute millivolts. Temperature in degrees C (Int. 1948). Reference junction at $0{ }^{\circ} \mathrm{C}$.

\begin{tabular}{|c|c|c|c|c|c|c|c|c|c|c|c|c|}
\hline Millivolts & .000 & .010 & .020 & .030 & .040 & .050 & .060 & .070 & .080 & .090 & .100 & Millivolts \\
\hline 0.000 & 0 & 3.2 & 6.3 & 9.4 & 12.5 & 15.5 & 18.6 & 21.6 & 24.5 & 27.5 & 30.4 & 0.000 \\
\hline .100 & 30.4 & 33.3 & 36.2 & 39.0 & 41.8 & 44.6 & 47.4 & 50.2 & 52.9 & 55.6 & 58.3 & .100 \\
\hline $\begin{array}{l}.200 \\
.300\end{array}$ & $\begin{array}{l}58.3 \\
84.0\end{array}$ & $\begin{array}{l}61.0 \\
86.5\end{array}$ & $\begin{array}{l}63.6 \\
89.0\end{array}$ & $\begin{array}{l}66.2 \\
91.5\end{array}$ & $\begin{array}{l}68.8 \\
94.0\end{array}$ & $\begin{array}{l}71.4 \\
96.5\end{array}$ & $\begin{array}{l}73.9 \\
98.9\end{array}$ & $\begin{array}{r}76.5 \\
101.4\end{array}$ & $\begin{array}{r}79.0 \\
103.8\end{array}$ & $\begin{array}{r}81.5 \\
106.2\end{array}$ & $\begin{array}{r}84.0 \\
108.6\end{array}$ & $\begin{array}{r}200 \\
300\end{array}$ \\
\hline & (2) & 00.0 & & & & & & & & & & \\
\hline .400 & 108. 6 & 111.0 & 113.3 & 115.7 & 118.0 & 120.3 & 122.7 & 125.0 & 127.3 & 129.5 & 131.8 & .400 \\
\hline .500 & 131.8 & 134. 1 & 136. 3 & 138.6 & 140.8 & 143.0 & 145. 2 & 147.4 & 149.6 & 151. 8 & 154. 0 & .500 \\
\hline .600 & 154.0 & 156. 2 & 158.3 & 160.5 & 162.6 & 164.8 & 166.9 & 169.1 & 171.2 & 173.3 & 175.4 & .600 \\
\hline .700 & 175.4 & 177.5 & 179.6 & 181.7 & 183.8 & 185.9 & 187.9 & 190.0 & 192.1 & 194.1 & 196. 2 & .700 \\
\hline .800 & 196. 2 & 198.3 & 200.3 & 202.4 & 204.4 & 206.5 & 208.5 & 210.5 & 212.6 & 214.6 & 216.6 & .800 \\
\hline .900 & 216.6 & 218.6 & 220.6 & 222.6 & 224.6 & 226.6 & 228.5 & 230.5 & 232.5 & 234.4 & 236.4 & .900 \\
\hline 1.000 & 236.4 & 238.4 & 240.3 & 242.3 & 244.2 & 246.1 & 248.1 & 250.0 & 251.9 & 253.9 & 255.8 & 1.000 \\
\hline 1.100 & 255.8 & 257.7 & 259.6 & 261.6 & 263.5 & 265.4 & 267.3 & 269.2 & 271.1 & 273. 0 & 274.9 & 1.100 \\
\hline 1. 200 & 274.9 & 276.8 & 278.7 & 280.6 & 282.5 & 284.3 & 286.2 & 288.1 & 290.0 & 291.8 & 293.7 & 1. 200 \\
\hline 1. 300 & 293.7 & 295.6 & 297.4 & 299.3 & 301.1 & 303.0 & 304.8 & 306.7 & 308.5 & 310.4 & 312.2 & 1. 300 \\
\hline 1. 400 & 312.2 & 314.0 & 315.9 & 317.7 & 319.5 & 321.3 & 323.1 & 325.0 & 326.8 & 328.6 & 330.4 & 1. 400 \\
\hline 1.500 & 330.4 & 332.2 & 334.1 & 335.9 & 337.7 & 339.5 & 341.3 & 343.2 & 345.0 & 346.8 & 348.6 & 1. 500 \\
\hline 1.600 & 348.6 & 350.4 & 352.2 & 354.0 & 355.7 & 357.5 & 359.3 & 361.1 & 362.8 & 364.6 & 366.4 & 1. 600 \\
\hline 1. 700 & 366.4 & 368.2 & 370.0 & 371.8 & 373.6 & 375.4 & 377.2 & 378.9 & 380.7 & 382.5 & 384.3 & 1. 700 \\
\hline 1.800 & 384. 3 & 386.1 & 387.8 & 389.6 & 391.3 & 393.1 & 394.8 & 396.6 & 398.3 & 400.1 & 401.8 & 1.800 \\
\hline 1.900 & 401.8 & 403.6 & 405.3 & 407.1 & 408.9 & 410.7 & 412.4 & 414.2 & 416.0 & 417.7 & 419.5 & 1.900 \\
\hline 2.000 & 419.5 & 421.2 & 423.0 & 424.7 & 426.5 & 428.2 & 430.0 & 431.7 & 433.4 & 435.2 & 436.9 & 2.000 \\
\hline 2.100 & 436.9 & 438.6 & 440.4 & 442.1 & 443.9 & 445.6 & 447.3 & 449.1 & 450.8 & 452.6 & 454. 3 & 2.100 \\
\hline 2. 200 & 454. 3 & 456.0 & 457.8 & 459.5 & 461.2 & 462.9 & 464.7 & 466.4 & 468.1 & 469.9 & 471.6 & 2. 200 \\
\hline 2. 300 & 471.6 & 473.3 & 475.1 & 476.8 & 478.6 & 480.3 & 482.0 & 483.8 & 485.5 & 487.3 & 489.0 & 2. 300 \\
\hline 2. 400 & 489. 0 & 490.7 & 492.4 & 494. 2 & 495.9 & 497.6 & 499. 3 & 501.0 & 502.8 & 504.5 & 506. 2 & 2. 400 \\
\hline 2. 500 & 506. 2 & 507.9 & 509.6 & 511.4 & 513.1 & 514.8 & 516.5 & 518.2 & 520.0 & 521.7 & 523.4 & 2.500 \\
\hline 2.600 & 523.4 & 525.1 & 526.8 & 528.5 & 530.2 & 531.9 & 533.7 & 535.4 & 537.1 & 538.8 & 540.5 & 2.600 \\
\hline 2. 700 & 540.5 & 542.2 & 543.9 & 545.7 & 547.4 & 549.1 & 550.8 & 552.5 & 554.3 & 556.0 & 557.7 & 2. 700 \\
\hline 2. 800 & 557.7 & 559.4 & 561.1 & 562.8 & 564.6 & 566.3 & 568.0 & 569.7 & 571.4 & 573.1 & 574.8 & 2.800 \\
\hline 2.900 & 574.8 & 576.5 & 578.2 & 579.9 & 581.6 & 583.3 & 585.0 & 586.7 & 588.4 & 590.1 & 591.8 & 2.900 \\
\hline 3.000 & 591.8 & 593.5 & 595.2 & 596.9 & 598.7 & 600.4 & 602.1 & 603.8 & 605.6 & 607.3 & 609.0 & 3.000 \\
\hline 3. 100 & 609.0 & 610.7 & 612.4 & 614. 2 & 615.9 & 617.6 & 619.3 & 621.0 & 622.8 & 624.5 & 626.2 & 3.100 \\
\hline 3. 200 & 626.2 & 627.9 & 629.7 & 631.4 & 633.1 & 634.9 & 636.6 & 638.3 & 640.1 & 641.8 & 643.5 & 3.200 \\
\hline 3. 300 & 643.5 & 645.2 & 646.9 & 648.6 & 650.3 & 652.0 & 653.7 & 655.4 & 657.1 & 658.8 & 660.5 & 3.300 \\
\hline 3. 400 & 660.5 & 662.2 & 663.9 & 665.6 & 667.4 & 669.1 & 670.8 & 672.5 & 674.2 & 676.0 & 677.7 & 3.400 \\
\hline 3. 500 & 677.7 & 679.4 & 681.1 & 682.9 & 684.6 & 686.3 & 688.1 & 689.8 & 691.5 & 693.3 & 695.0 & 3.500 \\
\hline 3.600 & 695.0 & 696.7 & 698.5 & 700.2 & 702.0 & 703.7 & 705.4 & 707.2 & 708.9 & 710.7 & 712.4 & 3.600 \\
\hline 3. 700 & 712.4 & 714.1 & 715.9 & 717. 6 & 719.4 & 721.1 & 722.8 & 724.6 & 726.3 & 728.1 & 729.8 & 3. 700 \\
\hline 3.800 & 729.8 & 731.5 & 733.3 & 735. 0 & 736.7 & 738.5 & 740.2 & 742.0 & 743.7 & 745.5 & 747.2 & 3.800 \\
\hline 3.900 & 747.2 & 749.0 & 750.7 & 752.5 & 754.2 & 756.0 & 757.7 & 759.5 & 761.3 & 763.0 & 764.8 & 3.900 \\
\hline 4. 000 & 764.8 & 766.6 & 768.3 & 770.1 & 771.9 & 773.6 & 775.4 & 777.2 & 779.0 & 780.7 & 782.5 & 4.000 \\
\hline 4. 100 & 782.5 & 784.3 & 786.0 & 787.8 & 789.5 & 791.3 & 793.1 & 794.8 & 796.6 & 798.3 & 800.1 & 4.100 \\
\hline 4. 200 & 800.1 & 801.9 & 803.7 & 805.4 & 807.2 & 809.0 & 810.8 & 812.6 & 814.3 & 816.1 & 817.9 & 4. 200 \\
\hline 4. 300 & 817.9 & 819.7 & 821.4 & 823.2 & 825.0 & 826.7 & 828.5 & 830.3 & 832.1 & 833.8 & 835.6 & 4. 300 \\
\hline 4. 400 & 835.6 & 837.4 & 839.2 & 840.9 & 842.7 & 844.5 & 846.3 & 848.0 & 849.8 & 851.6 & 853.4 & 4. 400 \\
\hline 4. 500 & 853.4 & 855.2 & 857.0 & 858.8 & 860.5 & 862.3 & 864.1 & 865.9 & 867.7 & 869.5 & 871.3 & 4. 500 \\
\hline 4. 600 & 871.3 & 873.1 & 874.9 & 876.7 & 878.5 & 880.3 & 882.2 & 884.0 & 885.8 & 887.6 & 889.4 & 4. 600 \\
\hline 4. 700 & 889.4 & 891. 2 & 893.0 & 894. 8 & 896.6 & 898.3 & 900.1 & 901. & 903 & 905.5 & 907.3 & 4. 700 \\
\hline 4. 800 & 907.3 & 909.1 & 910.9 & 912.7 & 914.5 & 916.3 & 918.1 & 919.9 & 921.8 & 923.6 & 925.4 & 4.800 \\
\hline 4.900 & 925.4 & 927.2 & 929.1 & 930.9 & 932.7 & 934.5 & 936.4 & 938.2 & 940.0 & 941.9 & 943.7 & 4.900 \\
\hline 5.000 & 943.7 & 945.5 & 947.3 & 949.1 & 950.9 & 952.7 & 954.6 & 956.4 & 958.2 & 960.0 & 961.8 & 5.000 \\
\hline 5. 100 & 961.8 & 963.6 & 965.5 & 967.3 & 969.1 & 971.0 & 972.8 & 974.7 & 976.5 & 978. & 980.2 & 5.100 \\
\hline $\begin{array}{l}5.200 \\
5.300\end{array}$ & $\begin{array}{l}980.2 \\
998.5\end{array}$ & $\begin{array}{r}982.0 \\
1000.3\end{array}$ & $\begin{array}{r}983.9 \\
1002.2\end{array}$ & $\begin{array}{r}985.7 \\
1004.0\end{array}$ & $\begin{array}{r}987.5 \\
1005.9\end{array}$ & $\begin{array}{r}989.3 \\
1007.7\end{array}$ & 991.2 & 993. & $\begin{array}{r}994.8 \\
1013.3\end{array}$ & 996. & $\begin{array}{r}998.5 \\
1017.0\end{array}$ & $\begin{array}{l}5.200 \\
5.300\end{array}$ \\
\hline & 998.0 & 1000.5 & 1002.2 & 1004. & 1005.9 & 007.7 & 009.6 & 1011.4 & 013 & 015.1 & 1017.0 & 5.300 \\
\hline 5. 400 & 1017.0 & 1018.8 & 1020.7 & 1022.5 & 1024. 4 & 1026. 2 & 1028.1 & 1029.9 & 1031. 7 & 1033. 6 & 1035.4 & 5.400 \\
\hline $\begin{array}{l}\text { 5. } 500 \\
5.600\end{array}$ & 1035.4 & 1037.2 & 1039.1 & 1040.9 & 1042. 7 & 1044.5 & 1046.4 & 1048. 2 & 1050.0 & 1051 & 1053.7 & 5. 500 \\
\hline 5. 600 & 1053.7 & 1055.5 & 1057.4 & 1059.2 & 1061.1 & 1062.9 & 1064.8 & 1066.6 & 1068.5 & 1070.3 & 1072.2 & 5.600 \\
\hline 5. 700 & 1072.2 & 1074.0 & 1075.9 & 1077.7 & 1079.6 & 1081.4 & 1083. 3 & 1085. 1 & 1087.0 & 1088.8 & 1090.7 & 5. 700 \\
\hline 5. 800 & 1090.7 & 1092.6 & 1094.4 & 1096.3 & 1098.1 & 1100.0 & 1101.9 & 1103.7 & 1105.6 & 1107.4 & 1109.3 & 5. 800 \\
\hline 5.900 & 1109.3 & 1111.2 & 1113.0 & 1114.9 & 1116.7 & 1118.6 & 1120.4 & 1122.3 & 1124.1 & 1126.0 & 1127.8 & 5.900 \\
\hline 6.000 & 1127.8 & 1129.6 & 1131.5 & 1133. 3 & 1135. 2 & 1137.0 & 1138.9 & 1140.7 & 1142.6 & 1144.4 & 1146.3 & 6.000 \\
\hline Millivolts & .000 & .010 & .020 & .030 & .040 & .050 & .060 & .070 & .080 & .090 & .100 & Millivolt \\
\hline
\end{tabular}


TABLE I-A. Forty percent iridium-60 percent rhodium versus iridium thermocouples-Continued Electromotive force in absolute millivolts. Temperature in degrees C (Int. 1948). Reference junction at $0{ }^{\circ} \mathrm{C}$.

\begin{tabular}{|c|c|c|c|c|c|c|c|c|c|c|c|c|}
\hline Millivolts & .000 & .010 & .020 & .030 & .040 & .050 & .060 & .070 & .080 & .090 & .100 & Millivolts \\
\hline 6.000 & 1127.8 & 1129.6 & 1131.5 & 1133.3 & 1135. 2 & 1137.0 & 1138.9 & 1140.7 & 1142.6 & 1144.4 & 1146.3 & 6.000 \\
\hline 6.100 & 1146. 3 & 1148.2 & 1150.0 & 1151.9 & 1153.8 & 1155.6 & 1157.5 & 1159.4 & 1161.3 & 1163.1 & 1165.0 & 6.100 \\
\hline 6. 200 & 1165.0 & 1166.9 & 1168.7 & 1170.6 & 1172.5 & 1174.4 & 1176.2 & 1178.1 & 1180.0 & 1181.8 & 1183.7 & 6. 200 \\
\hline & & & 1187.4 & 1189.3 & 1191.1 & 1193.0 & 1194.9 & 1196.7 & 1198.6 & 1200.4 & 1202.3 & \\
\hline 6.400 & 1202.3 & 1204.2 & 1206.0 & 1207.9 & 1209.7 & 1211.6 & 1213.4 & 1215.3 & 1217.2 & 1219.0 & 1220.9 & 6.400 \\
\hline 6.500 & 1220.9 & 1222.8 & 1224.7 & 1226.6 & 1228.5 & 1230.3 & 1222.2 & 1234.1 & 1236. 0 & 1237.9 & 1239.8 & 6.500 \\
\hline 6.600 & 1239.8 & 1241.7 & 1243.5 & 1245.4 & 1247.3 & 1249.2 & 1251.0 & 1252.9 & 1254.8 & 1256.6 & 1258.5 & 6.600 \\
\hline 6.700 & 1258.5 & 1260.4 & 1262.2 & 1264.1 & 1266.0 & 1267.8 & 1269.7 & 1271.6 & 1273.5 & 1275.3 & 1277.2 & 6.700 \\
\hline 6.800 & 1277.2 & 1279.1 & 1280.9 & 1282.8 & 1284.7 & 1286.5 & 1288.4 & 1290.3 & 1292. 2 & 1294.0 & 1295.9 & 6.800 \\
\hline 6.900 & 1295.9 & 1297.8 & 1299.7 & 1301.5 & 1303.4 & 1305.2 & 1307.1 & 1309.0 & 1310.9 & 1312.7 & 1314.6 & 6.900 \\
\hline 7.000 & 1314.6 & 1316.5 & 1318. 3 & 1320.2 & 1322.1 & 1324.0 & 1325.8 & 1327.7 & 1329.6 & 1331.4 & 1333. 3 & 7.000 \\
\hline 7. 100 & 1333. 3 & 1335. 2 & 1337.0 & 1338.9 & 1340.7 & 1342.6 & 1344. 4 & 1346. 3 & 1348. 1 & 1350.0 & 1351.8 & 7.100 \\
\hline 7. 200 & 1351.8 & 1353. 7 & 1355.5 & 1357.4 & 1359.2 & 1361.1 & 1363.0 & 1364.8 & 1366. 7 & 1368.5 & 1370.4 & 7. 200 \\
\hline 7.300 & 1370.4 & 1372.2 & 1374.1 & 1375.9 & 1377.7 & 1379.6 & 1381.4 & 1383.2 & 1385.0 & 1386.9 & 1388.7 & 7.300 \\
\hline 7.400 & 1388.7 & 1390.5 & 1392.4 & 1394.2 & 1396.0 & 1397.8 & 1399. 7 & 1401.5 & 1403. 3 & 1405.2 & 1407.0 & 7.400 \\
\hline 7. 500 & 1407.0 & 1408.8 & 1410.7 & 1412.5 & 1414.4 & 1416. 2 & 1418. 1 & 1419.9 & 1421.7 & 1423.6 & 1425. 4 & 7.500 \\
\hline 7.600 & 1425.4 & 1427.2 & 1429.0 & 1430.8 & 1432.7 & 1434.5 & 1436.3 & 1438.1 & 1439.9 & 1441.7 & 1443.5 & 7.600 \\
\hline 7. 700 & 1443.5 & 1445.3 & 1447.1 & 1448.9 & 1450.7 & 1452.5 & 1454.4 & 1456.2 & 1458.0 & 1459.8 & 1461.6 & 7. 700 \\
\hline 7.800 & 1461.6 & 1463.4 & 1465. 2 & 1467.0 & 1468.9 & 1470.7 & 1472.5 & 1474.3 & 1476.1 & 1477.9 & 1479.7 & 7.800 \\
\hline 7.900 & 1479.7 & 1481.5 & 1483.3 & 1485.1 & 1486.9 & 1488.7 & 1490.5 & 1492.2 & 1494.0 & 1495.8 & 1497.6 & 7.900 \\
\hline 8.000 & 1497.6 & 1499.4 & 1501.2 & 1503.0 & 1504.7 & 1506.5 & 1508.3 & 1510.1 & 1511.9 & 1513.6 & 1515.4 & 8.000 \\
\hline 8.100 & 1515.4 & 1517.2 & 1518.9 & 1520.7 & 1522.4 & 1524. 2 & 1525.9 & 1527.7 & 1529.4 & 1531.2 & 1532.9 & 8.100 \\
\hline 8.200 & 1532.9 & 1534.6 & 1536.4 & 1538.1 & 1539.9 & 1541. 6 & 1543.3 & 1545.1 & 1546.8 & 1548.6 & 1550.3 & 8.200 \\
\hline 8.300 & 1550.3 & 1552.0 & 1553.8 & 1555.5 & 1557.2 & 1559.0 & 1560.7 & 1562.5 & 1564.2 & 1566.0 & 1567. 7 & 8.300 \\
\hline 8. 400 & 1567.7 & 1569.5 & 1571. 2 & 1573.0 & 1574. 7 & 1576.5 & 1578.3 & 1580.0 & 1581.8 & 1583.5 & 1585. 3 & 8. 400 \\
\hline 8.500 & 1585.3 & 1587.0 & 1588.8 & 1590.5 & 1592.3 & 1594.0 & 1595.8 & 1597.5 & 1599. 3 & 1601.0 & 1602.8 & 8.500 \\
\hline 8.600 & 1602.8 & 1604.6 & 1606.3 & 1608.1 & 1609.9 & 1611.6 & 1613.4 & 1615.2 & 1617.0 & 1618.7 & 1620.5 & 8.600 \\
\hline 8. 700 & 1620.5 & 1622.3 & 1624.0 & 1625.8 & 1627.5 & 1629.3 & 1631.1 & 1632.8 & 1634.6 & 1636. 3 & 1638.1 & 8. 700 \\
\hline 8.800 & 1638.1 & 1639.9 & 1641.6 & 1643.4 & 1645. 2 & 1646.9 & 1648.7 & 1650.4 & 1652.2 & 1653.9 & 1655.7 & 8.800 \\
\hline 8.900 & 1655.7 & 1657.4 & 1659.2 & 1660.9 & 1662.6 & 1664.4 & 1666.1 & 1667.8 & 1669.5 & 1671.3 & 1673.0 & 8.900 \\
\hline 9.000 & 1673.0 & 1674.7 & 1676.5 & 1678.2 & 1680.0 & 1681.7 & 1683.5 & 1685.2 & 1686.9 & 1688.7 & 1690.4 & 9.000 \\
\hline 9. 100 & 1690.4 & 1692.1 & 1693.8 & 1695.5 & 1697.2 & 1699.0 & 1700.7 & 1702 & 1704.1 & 1705.8 & 1707.5 & 9.100 \\
\hline 9.200 & 1707.5 & 1709.2 & 1711.0 & 1712.7 & 1714.4 & 1716. 2 & 1717. 9 & 1719.6 & 1721.3 & 1723.1 & 1724.8 & 9. 200 \\
\hline 9.300 & 1724.8 & 1726.5 & 1728.2 & 1729.9 & 1731.6 & 1733.3 & 1735.0 & 1736.7 & 1738.4 & 1740.1 & 1741.8 & 9.300 \\
\hline 9.400 & 1741.8 & 1743.5 & 1745.2 & 1746.8 & 1748.5 & 1750.2 & 1751.9 & 1753.6 & 1755.2 & 1756.9 & 1758.6 & 9. 400 \\
\hline 9.500 & 1758.6 & 1760.3 & 1762.0 & 1763.7 & 1765.4 & 1767.0 & 1768.7 & 1770.4 & 1772.1 & 1773.8 & 1775.5 & 9.500 \\
\hline 9.600 & 1775.5 & 1777.2 & 1778.9 & 1780.5 & 1782.2 & 1783.9 & 1785.6 & 1787.3 & 1788.9 & 1790.6 & 1792.3 & 9.600 \\
\hline 9.700 & 1792. 3 & 1794. & 1795. 7 & 1797.4 & 1799.0 & 1800.7 & 1802.4 & 1804 & 1805.8 & 1807.4 & 1809.1 & 9. 700 \\
\hline 9.800 & 1809.1 & 1810 . & 1812.4 & 1814.1 & 1815.7 & 1817.4 & 1819.0 & 1820.7 & 1822.3 & 1824.0 & 1825.6 & 9.800 \\
\hline 9.900 & 1825.6 & 1827.2 & 1828.9 & 1830.5 & 1832.2 & 1833.8 & 1835.5 & 1837.1 & 1838.8 & 1840.4 & 1842.1 & 9.900 \\
\hline 10.000 & 1842.1 & 1843.8 & 1845.4 & 1847.1 & 1848.7 & 1850.4 & 1852.0 & 1853.7 & 1855.3 & 1857.0 & 1858.6 & 10.000 \\
\hline 10.100 & 1858.6 & 1860.2 & 1861.9 & 1863.5 & 1865. 2 & 1866.8 & 1868.5 & 1870.1 & 1871.7 & 1873.4 & 1875.0 & 10.100 \\
\hline 10. 200 & 1875.0 & 1876.6 & 1878.3 & 1879.9 & 1881.5 & 1883.2 & 1884.8 & 1886.4 & 1888.1 & 1889.7 & 1891. 3 & 10. 200 \\
\hline 10.300 & 1891.3 & 1892.9 & 1894.5 & 1896.1 & 1897.7 & 1899.3 & 1900.9 & 1902.4 & 1904.0 & 1905.6 & 1907.2 & 10.300 \\
\hline 10.400 & 1907. 2 & 1908.8 & 1910.4 & 1912.0 & 1913.6 & 1915.1 & 1916. 7 & 1918.3 & 1919.9 & 1921.5 & 1923. 1 & 10.400 \\
\hline 10.500 & 1923. 1 & 1924.7 & 1926. 3 & 1927.9 & 1929.5 & 1931.1 & 1932.7 & 1934. 2 & 1935.8 & 1937.4 & 1939.0 & 10.500 \\
\hline 10.600 & 1939.0 & 1940.6 & 1942.1 & 1943.7 & 1945.3 & 1946.8 & 1948.4 & 1949.9 & 1951.5 & 1953.1 & 1954.6 & 10.600 \\
\hline 10. 700 & 1954.6 & 56.2 & 1 & 959 & 1960. & 1962.3 & 1963 & 19 & 19 & 19 & 0 & 10. 700 \\
\hline 10.800 & 1970.0 & 1971.5 & 1973.0 & 1974.4 & 1975.9 & 1977.4 & 197 & 198 & 1981.9 & 198 & 198 & 10.800 \\
\hline 10.900 & 1984.9 & 1986.5 & 1988.0 & 1989.6 & 1991.2 & 1992.8 & 1994.4 & 1995.9 & 1997.5 & 1999.1 & 2000.7 & 10.900 \\
\hline 11.000 & 2000.7 & 2002.2 & 2003.8 & 2005.3 & 2006.9 & 2008.4 & 2009.9 & 2011.4 & 2013.0 & 2014.5 & 2016.0 & 11.000 \\
\hline 11.100 & 2016. 0 & 2017.5 & 2019. 1 & 2020.6 & 2022.1 & 2023.7 & 2025. 2 & 2026.7 & 2028.3 & 2029.8 & 2031. 3 & 11. 100 \\
\hline 11. 200 & 2031. 3 & 2032.8 & 2034. 3 & 2035.8 & 2037.3 & 2038.8 & 2040.3 & 2041.8 & 2043. 3 & 2044.8 & 2046. 3 & 11. 200 \\
\hline 11.300 & 2046.3 & 2047.8 & 2049.3 & 2050.9 & 2052.4 & 2053.9 & 2055.5 & 2057.0 & 2058.5 & 2060.1 & 2061.6 & 11. 300 \\
\hline 11. 400 & 2061.6 & 2063.1 & 2064.7 & 2066. 2 & 2067.7 & 2069.2 & 2070.7 & 2072.3 & 2073.8 & 2075.3 & 2076.8 & 11. 400 \\
\hline 11 & 2076.8 & 2078.3 & 2079.8 & 2081.3 & 2082.8 & 2084.3 & 2085.8 & 2087.3 & 2088.8 & 2090.3 & 2091.8 & 11. 500 \\
\hline 1. 600 & 2091.8 & 2093. 3 & 2094.8 & & & & & & & & & \\
\hline Millivolts & .000 & .010 & .020 & .030 & .040 & .050 & .060 & .070 & .080 & .090 & .100 & Millivolts \\
\hline
\end{tabular}


TABLE II-A. Forty percent iridium-60 percent rhodium versus iridium thermocouples

Electromotive force in absolute millivolts. Temperature in degrees C (Int. 1948). Reference junction at $0{ }^{\circ} \mathrm{C}$.

\begin{tabular}{|c|c|c|c|c|c|c|c|c|c|c|c|c|}
\hline${ }^{\circ} \mathrm{C}$ & 0 & 10 & 20 & 30 & 40 & 50 & 60 & 70 & 80 & 90 & 100 & ${ }^{\circ} \mathrm{C}$ \\
\hline $\begin{array}{c}0 \\
100 \\
200 \\
300 \\
400\end{array}$ & $\begin{array}{r}0.000 \\
.365 \\
.818 \\
1.334 \\
1.889\end{array}$ & $\begin{array}{r}0.032 \\
.406 \\
.868 \\
1.388 \\
1.946\end{array}$ & $\begin{array}{r}0.064 \\
.449 \\
.917 \\
1.443 \\
2.003\end{array}$ & $\begin{array}{r}0.098 \\
.492 \\
.968 \\
1.498 \\
2.060\end{array}$ & $\begin{array}{r}0.134 \\
.536 \\
1.019 \\
1.553 \\
2.118\end{array}$ & $\begin{array}{r}0.170 \\
.581 \\
1.070 \\
1.608 \\
2.175\end{array}$ & $\begin{array}{r}0.206 \\
.627 \\
1.122 \\
1.664 \\
2.233\end{array}$ & $\begin{array}{l}0.245 \\
.674 \\
1.174 \\
1.720 \\
2.291\end{array}$ & $\begin{array}{r}0.284 \\
.722 \\
1.227 \\
1.776 \\
2.348\end{array}$ & $\begin{array}{r}0.324 \\
.770 \\
1.280 \\
1.832 \\
2.406\end{array}$ & $\begin{array}{r}0.365 \\
.818 \\
1.334 \\
1.889 \\
2.464\end{array}$ & $\begin{array}{c}0 \\
100 \\
200 \\
300 \\
400\end{array}$ \\
\hline $\begin{array}{l}500 \\
600 \\
700 \\
800 \\
900\end{array}$ & $\begin{array}{l}2.464 \\
\text { 3. } 047 \\
3.628 \\
4.199 \\
4.759\end{array}$ & $\begin{array}{l}2.522 \\
3.106 \\
3.686 \\
4.256 \\
4.815\end{array}$ & $\begin{array}{l}2.580 \\
3.164 \\
3.744 \\
4.312 \\
4.870\end{array}$ & $\begin{array}{l}\text { 2. } 639 \\
\text { 3. } 222 \\
\text { 3. } 801 \\
\text { 4. } 368 \\
\text { 4. } 925\end{array}$ & $\begin{array}{l}2.697 \\
3.280 \\
3.859 \\
4.424 \\
4.980\end{array}$ & $\begin{array}{l}\text { 2. } 755 \\
\text { 3. } 339 \\
\text { 3. } 916 \\
\text { 4. } 480 \\
\text { 5. } 035\end{array}$ & $\begin{array}{l}2.814 \\
3.397 \\
3.973 \\
\text { 4. } 537 \\
5.090\end{array}$ & $\begin{array}{l}\text { 2. } 872 \\
\text { 3. } 455 \\
\text { 4. } 030 \\
\text { 4. } 593 \\
\text { 5. } 144\end{array}$ & $\begin{array}{l}2.930 \\
3.513 \\
4.086 \\
4.648 \\
5.199\end{array}$ & $\begin{array}{l}\text { 2. } 989 \\
3.571 \\
4.143 \\
4.703 \\
5.254\end{array}$ & $\begin{array}{l}\text { 3. } 047 \\
\text { 3. } 628 \\
\text { 4. } 199 \\
\text { 4. } 759 \\
\text { 5. } 308\end{array}$ & $\begin{array}{l}500 \\
600 \\
700 \\
800 \\
900\end{array}$ \\
\hline $\begin{array}{l}1000 \\
1100 \\
1200 \\
1300 \\
1400\end{array}$ & $\begin{array}{l}5.308 \\
5.850 \\
6.388 \\
6.922 \\
7.461\end{array}$ & $\begin{array}{l}\text { 5. } 362 \\
5.904 \\
6.441 \\
6.976 \\
7.516\end{array}$ & $\begin{array}{l}5.417 \\
5.958 \\
6.493 \\
7.029 \\
7.571\end{array}$ & $\begin{array}{l}5.471 \\
6.012 \\
6.548 \\
7.082 \\
7.626\end{array}$ & $\begin{array}{l}\text { 5. } 525 \\
6.066 \\
6.601 \\
7.136 \\
7.681\end{array}$ & $\begin{array}{l}5.580 \\
6.120 \\
6.654 \\
7.190 \\
7.736\end{array}$ & $\begin{array}{l}5.634 \\
6.173 \\
6.708 \\
7.244 \\
7.791\end{array}$ & $\begin{array}{l}\text { 5. } 688 \\
\text { 6. } 227 \\
\text { 6. } 761 \\
\text { 7. } 298 \\
\text { 7. } 847\end{array}$ & $\begin{array}{l}\text { 5. } 742 \\
6.280 \\
6.815 \\
7.352 \\
7.902\end{array}$ & $\begin{array}{l}\text { 5. } 796 \\
6.334 \\
6.869 \\
7.407 \\
7.957\end{array}$ & $\begin{array}{l}\text { 5. } 850 \\
6.388 \\
6.922 \\
7.461 \\
\text { 8. } 013\end{array}$ & $\begin{array}{l}1000 \\
1100 \\
1200 \\
1300 \\
1400\end{array}$ \\
\hline $\begin{array}{l}1500 \\
1600 \\
1700 \\
1800 \\
1900\end{array}$ & $\begin{array}{r}8.013 \\
8.583 \\
9.156 \\
9.745 \\
10.355\end{array}$ & $\begin{array}{r}8.070 \\
8.640 \\
9.214 \\
9.805 \\
10.417\end{array}$ & $\begin{array}{r}8.127 \\
8.697 \\
9.272 \\
9.866 \\
10.480\end{array}$ & $\begin{array}{r}8.183 \\
8.754 \\
9.331 \\
9.926 \\
10.543\end{array}$ & $\begin{array}{r}8.241 \\
8.811 \\
9.390 \\
9.987 \\
10.606\end{array}$ & $\begin{array}{r}8.298 \\
8.868 \\
9.449 \\
10.048 \\
10.670\end{array}$ & $\begin{array}{r}8.356 \\
8.925 \\
9.508 \\
10.108 \\
10.735\end{array}$ & $\begin{array}{r}8.413 \\
8.983 \\
9.567 \\
10.169 \\
10.800\end{array}$ & $\begin{array}{r}8.470 \\
9.040 \\
9.627 \\
10.230 \\
10.865\end{array}$ & $\begin{array}{r}8.526 \\
9.098 \\
9.686 \\
10.292 \\
10.930\end{array}$ & $\begin{array}{r}8.583 \\
9.156 \\
9.745 \\
10.355 \\
10.995\end{array}$ & $\begin{array}{l}1500 \\
1600 \\
1700 \\
1800 \\
1900\end{array}$ \\
\hline $\begin{array}{l}2000 \\
2100\end{array}$ & $\begin{array}{l}10.995 \\
11.654\end{array}$ & 11. 061 & 11.126 & 11.192 & 11.258 & 11.324 & 11. 389 & 11. 455 & 11. 521 & 11. 588 & 11. 654 & 2000 \\
\hline${ }^{\circ} \mathrm{C}$ & 0 & 10 & 20 & 30 & 40 & 50 & 60 & 70 & 80 & 90 & 100 & ${ }^{\circ} \mathrm{C}$ \\
\hline
\end{tabular}

TABLE III-A. Forty percent iridium-60 percent rhodium versus iridium thermocouples

Electromotive force in absolute millivolts. Temperature in degrees F (Int. 1948). Reference junction at $32{ }^{\circ} \mathrm{F}$.

\begin{tabular}{|c|c|c|c|c|c|c|c|c|c|c|c|c|}
\hline Millivolts & .000 & 010 & .020 & .030 & .040 & .050 & 060 & .070 & .080 & .090 & .100 & Millivolts \\
\hline 0.000 & 32.0 & 37.7 & 43.4 & 49.0 & 54.5 & 60.0 & 65.5 & 70.9 & 76.2 & 81.5 & 86.8 & 0.000 \\
\hline $\begin{array}{l}.100 \\
.200 \\
.300\end{array}$ & $\begin{array}{r}86.8 \\
137.0 \\
183.2\end{array}$ & $\begin{array}{r}92.0 \\
141.8 \\
187.7\end{array}$ & $\begin{array}{r}97.2 \\
146.5 \\
192.2\end{array}$ & $\begin{array}{l}102.3 \\
151.2 \\
196.7\end{array}$ & $\begin{array}{l}107.4 \\
155.8 \\
201.1\end{array}$ & $\begin{array}{l}112.4 \\
160.5 \\
205.6\end{array}$ & $\begin{array}{l}117.4 \\
165.1 \\
210.0\end{array}$ & $\begin{array}{l}122.4 \\
169.6 \\
214.4\end{array}$ & $\begin{array}{l}127.3 \\
174.2 \\
218.7\end{array}$ & $\begin{array}{l}132.2 \\
178.7 \\
223.1\end{array}$ & $\begin{array}{l}137.0 \\
183.2 \\
227.4\end{array}$ & $\begin{array}{l}.100 \\
.200 \\
.300\end{array}$ \\
\hline $\begin{array}{l}.400 \\
.500 \\
.600\end{array}$ & $\begin{array}{l}227.4 \\
269.2 \\
309.2\end{array}$ & $\begin{array}{l}231.7 \\
273.3 \\
313.1\end{array}$ & $\begin{array}{l}235.9 \\
277.3 \\
317.0\end{array}$ & $\begin{array}{l}240.2 \\
281.4 \\
320.9\end{array}$ & $\begin{array}{l}244.4 \\
285.4 \\
324.8\end{array}$ & $\begin{array}{l}248.6 \\
289.4 \\
328.7\end{array}$ & $\begin{array}{l}252.7 \\
293.4 \\
332.5\end{array}$ & $\begin{array}{l}256.9 \\
297.4 \\
336.4\end{array}$ & $\begin{array}{l}261.0 \\
301.3 \\
340.2\end{array}$ & $\begin{array}{l}265.1 \\
305.3 \\
344.0\end{array}$ & $\begin{array}{l}269.2 \\
309.2 \\
347.8\end{array}$ & $\begin{array}{l}.400 \\
.500 \\
.600\end{array}$ \\
\hline $\begin{array}{l}.700 \\
.800 \\
.900\end{array}$ & $\begin{array}{l}347.8 \\
385.2 \\
421.8\end{array}$ & $\begin{array}{l}351.6 \\
388.9 \\
425.4\end{array}$ & $\begin{array}{l}355.4 \\
392.6 \\
429.0\end{array}$ & $\begin{array}{l}359.1 \\
396.3 \\
432.6\end{array}$ & $\begin{array}{l}362.9 \\
399.9 \\
436.2\end{array}$ & $\begin{array}{l}366.6 \\
403.6 \\
439.8\end{array}$ & $\begin{array}{l}370.4 \\
407.3 \\
443.3\end{array}$ & $\begin{array}{l}374.1 \\
410.9 \\
446.9\end{array}$ & $\begin{array}{l}377.8 \\
414.5 \\
450.4\end{array}$ & $\begin{array}{l}381.5 \\
418.2 \\
454.0\end{array}$ & $\begin{array}{l}385.2 \\
421.8 \\
457.5\end{array}$ & $\begin{array}{l}.700 \\
.800 \\
.900\end{array}$ \\
\hline 1.000 & 457.5 & 461.0 & 464.5 & 468.0 & 471.5 & 475.0 & 478.5 & 482.0 & 485.5 & 488.9 & 492.4 & 1.000 \\
\hline $\begin{array}{l}1.100 \\
1.200 \\
1.300\end{array}$ & $\begin{array}{l}492.4 \\
526.9 \\
560.7\end{array}$ & $\begin{array}{l}495.9 \\
530.3 \\
564.0\end{array}$ & $\begin{array}{l}499.3 \\
533.7 \\
567.4\end{array}$ & $\begin{array}{l}502.8 \\
537.1 \\
570.7\end{array}$ & $\begin{array}{l}506.3 \\
540.5 \\
574.1\end{array}$ & $\begin{array}{l}509.7 \\
543.9 \\
577.4\end{array}$ & $\begin{array}{l}513.2 \\
547.3 \\
580.7\end{array}$ & $\begin{array}{l}516.6 \\
550.6 \\
584.1\end{array}$ & $\begin{array}{l}520.0 \\
554.0 \\
587.4\end{array}$ & $\begin{array}{l}523.5 \\
557.3 \\
590.7\end{array}$ & $\begin{array}{l}526.9 \\
560.7 \\
594.0\end{array}$ & $\begin{array}{l}1.100 \\
1.200 \\
1.300\end{array}$ \\
\hline $\begin{array}{l}1.400 \\
1.500 \\
1.600\end{array}$ & $\begin{array}{l}594.0 \\
626.7 \\
659.4\end{array}$ & $\begin{array}{l}597.3 \\
630.0 \\
662.6\end{array}$ & $\begin{array}{l}600.6 \\
633.3 \\
665.9\end{array}$ & $\begin{array}{l}603.8 \\
636.5 \\
669.1\end{array}$ & $\begin{array}{l}607.1 \\
639.8 \\
672.3\end{array}$ & $\begin{array}{l}610.4 \\
643.1 \\
675.5\end{array}$ & $\begin{array}{l}613.7 \\
646.4 \\
678.7\end{array}$ & $\begin{array}{l}616.9 \\
649.6 \\
682.0\end{array}$ & $\begin{array}{l}620.2 \\
652.9 \\
685.2\end{array}$ & $\begin{array}{l}623.4 \\
656.1 \\
688.4\end{array}$ & $\begin{array}{l}626.7 \\
659.4 \\
691.6\end{array}$ & $\begin{array}{l}1.400 \\
1.500 \\
1.600\end{array}$ \\
\hline $\begin{array}{l}1.700 \\
1.800 \\
1.900\end{array}$ & $\begin{array}{l}691.6 \\
723.8 \\
755.3\end{array}$ & $\begin{array}{l}694.8 \\
727.0 \\
758.5\end{array}$ & $\begin{array}{l}698.1 \\
730.1 \\
761.7\end{array}$ & $\begin{array}{l}701.3 \\
733.3 \\
764.8\end{array}$ & $\begin{array}{l}704.5 \\
736.4 \\
768.0\end{array}$ & $\begin{array}{l}707.7 \\
739.6 \\
771.2\end{array}$ & $\begin{array}{l}711.0 \\
742.7 \\
774.4\end{array}$ & $\begin{array}{l}714.2 \\
745.9 \\
777.6\end{array}$ & $\begin{array}{l}717.4 \\
749.0 \\
780.8\end{array}$ & $\begin{array}{l}720.6 \\
752.2 \\
783.9\end{array}$ & $\begin{array}{l}723.8 \\
755.3 \\
787.1\end{array}$ & $\begin{array}{l}1.700 \\
1.800 \\
1.900\end{array}$ \\
\hline 2.000 & 787.1 & 790.2 & 793.4 & 796.5 & 799.7 & 802.8 & 805.9 & 809.0 & 812.2 & 815.3 & 818.4 & 2. 000 \\
\hline $\begin{array}{l}2.100 \\
2.200 \\
2.300\end{array}$ & $\begin{array}{l}818.4 \\
849.7 \\
880.9\end{array}$ & $\begin{array}{l}821.5 \\
852.8 \\
884.0\end{array}$ & $\begin{array}{l}824.7 \\
855.9 \\
887.2\end{array}$ & $\begin{array}{l}827.8 \\
859.1 \\
890.3\end{array}$ & $\begin{array}{l}830.9 \\
862.2 \\
893.4\end{array}$ & $\begin{array}{l}834.1 \\
865.3 \\
896.6\end{array}$ & $\begin{array}{l}837.2 \\
868.4 \\
899.7\end{array}$ & $\begin{array}{l}840.3 \\
871.5 \\
902.8\end{array}$ & $\begin{array}{l}843.4 \\
874.7 \\
906.0\end{array}$ & $\begin{array}{l}846.6 \\
877.8 \\
909.1\end{array}$ & $\begin{array}{l}849.7 \\
880.9 \\
912.2\end{array}$ & $\begin{array}{l}2.100 \\
2.200 \\
2.300\end{array}$ \\
\hline $\begin{array}{l}2.400 \\
2.500 \\
2.600\end{array}$ & $\begin{array}{l}912.2 \\
943.1 \\
974.1\end{array}$ & $\begin{array}{l}915.3 \\
946.2 \\
977.2\end{array}$ & $\begin{array}{l}918.4 \\
949.3 \\
980.3\end{array}$ & $\begin{array}{l}921.5 \\
952.4 \\
983.3\end{array}$ & $\begin{array}{l}924.6 \\
955.5 \\
986.4\end{array}$ & $\begin{array}{l}927.7 \\
958.6 \\
989.5\end{array}$ & $\begin{array}{l}930.8 \\
961.7 \\
992.6\end{array}$ & $\begin{array}{l}933.8 \\
964.8 \\
995.7\end{array}$ & $\begin{array}{l}936.9 \\
967.9 \\
998.7\end{array}$ & $\begin{array}{r}940.0 \\
971.0 \\
1001.8\end{array}$ & $\begin{array}{r}943.1 \\
974.1 \\
1004.9\end{array}$ & $\begin{array}{l}2.400 \\
2.500 \\
2.600\end{array}$ \\
\hline $\begin{array}{l}2.700 \\
2.800 \\
2.900\end{array}$ & $\begin{array}{l}1004.9 \\
1035.8 \\
1066.7\end{array}$ & $\begin{array}{l}1008.0 \\
1038.9 \\
1069.8\end{array}$ & $\begin{array}{l}1011.1 \\
1042.0 \\
1072.8\end{array}$ & $\begin{array}{l}1014.2 \\
1045.1 \\
1075.9\end{array}$ & $\begin{array}{l}1017.3 \\
1048.2 \\
1078.9\end{array}$ & $\begin{array}{l}1020.3 \\
1051.3 \\
1082.0\end{array}$ & $\begin{array}{l}1023.4 \\
1054.4 \\
1085.1\end{array}$ & $\begin{array}{l}1026.5 \\
1057.4 \\
1088.1\end{array}$ & $\begin{array}{l}1029.6 \\
1060.5 \\
1091.2\end{array}$ & $\begin{array}{l}1032.7 \\
1063.6 \\
1094.2\end{array}$ & $\begin{array}{l}1035.8 \\
1066.7 \\
1097.3\end{array}$ & $\begin{array}{l}2.700 \\
2.800 \\
2.900\end{array}$ \\
\hline 3.000 & 1097.3 & 1100.4 & 1103.4 & 1106.5 & 1109.6 & 1112.7 & 1115.8 & 1118.9 & 1122.0 & 1125.1 & 1128.2 & 3.000 \\
\hline $\begin{array}{l}3.100 \\
3.200 \\
3.300\end{array}$ & $\begin{array}{l}1128.2 \\
1159.2 \\
1190.3\end{array}$ & $\begin{array}{l}1131.3 \\
1162.3 \\
1193.4\end{array}$ & $\begin{array}{l}1134.4 \\
1165.4 \\
1196.4\end{array}$ & $\begin{array}{l}1137.5 \\
1168.5 \\
1199.5\end{array}$ & $\begin{array}{l}1140.6 \\
1171.6 \\
1202.5\end{array}$ & $\begin{array}{l}1143.7 \\
1174.7 \\
1205.6\end{array}$ & $\begin{array}{l}1146.8 \\
1177.8 \\
1208.6\end{array}$ & $\begin{array}{l}1149.9 \\
1180.9 \\
1211.7\end{array}$ & $\begin{array}{l}1153.0 \\
1184.1 \\
1214.8\end{array}$ & $\begin{array}{l}1156.1 \\
1187.2 \\
1217.8\end{array}$ & $\begin{array}{l}1159.2 \\
1190.3 \\
1220.9\end{array}$ & $\begin{array}{l}\text { 3. } 100 \\
\text { 3. } 200 \\
\text { 3. } 300\end{array}$ \\
\hline $\begin{array}{l}3.400 \\
3.500 \\
3.600\end{array}$ & $\begin{array}{l}1220.9 \\
1251.9 \\
1283.1\end{array}$ & $\begin{array}{l}1224.0 \\
1255.0 \\
1286.2\end{array}$ & $\begin{array}{l}1227.1 \\
1258.1 \\
1289.4\end{array}$ & $\begin{array}{l}1230.2 \\
1261.2 \\
1292.5\end{array}$ & $\begin{array}{l}1233.3 \\
1264.4 \\
1295.6\end{array}$ & $\begin{array}{l}1236.4 \\
1267.5 \\
1298.7\end{array}$ & $\begin{array}{l}1239.5 \\
1270.6 \\
1301.9\end{array}$ & $\begin{array}{l}1242.6 \\
1273.7 \\
1305.0\end{array}$ & $\begin{array}{l}1245.7 \\
1276.8 \\
1308.1\end{array}$ & $\begin{array}{l}1248.8 \\
1280.0 \\
1311.3\end{array}$ & $\begin{array}{l}1251.9 \\
1283.1 \\
1314.4\end{array}$ & $\begin{array}{l}3.400 \\
3.500 \\
3.600\end{array}$ \\
\hline $\begin{array}{l}3.700 \\
\text { 3. } 800 \\
3.900\end{array}$ & $\begin{array}{l}1314.4 \\
1345.6 \\
1376.9\end{array}$ & $\begin{array}{l}1317.5 \\
1348.7 \\
1380.1\end{array}$ & $\begin{array}{l}1320.6 \\
1351.8 \\
1383.2\end{array}$ & $\begin{array}{l}1323.8 \\
1355.0 \\
1386.4\end{array}$ & $\begin{array}{l}1326.9 \\
1358.1 \\
1389.6\end{array}$ & $\begin{array}{l}1330.0 \\
1361.2 \\
1392.8\end{array}$ & $\begin{array}{l}1333.1 \\
1364.3 \\
1395.9\end{array}$ & $\begin{array}{l}1336.2 \\
1367.5 \\
1399.1\end{array}$ & $\begin{array}{l}1339.4 \\
1370.6 \\
1402.3\end{array}$ & $\begin{array}{l}1342.5 \\
1373.8 \\
1405.5\end{array}$ & $\begin{array}{l}1345.6 \\
1376.9 \\
1408.7\end{array}$ & $\begin{array}{l}3.700 \\
3.800 \\
3.900\end{array}$ \\
\hline Millivolts & .000 & .010 & .020 & .030 & .040 & .050 & .060 & .070 & .080 & .090 & .100 & Millivolts \\
\hline
\end{tabular}


TABle III-A. Forty percent iridium-60 percent rhodium versus iridium thermocouples - Continued

Electromotive force in absolute millivolts. Temperature in degrees F (Int. 1948). Reference junction at $32{ }^{\circ} \mathrm{F}$.

\begin{tabular}{|c|c|c|c|c|c|c|c|c|c|c|c|c|}
\hline Millivolts & .000 & .010 & .020 & .030 & .040 & .050 & .060 & .070 & .080 & .090 & .100 & Millivolts \\
\hline 4. 000 & 1408.7 & 1411.9 & 1415.1 & 1418. 3 & 1421.5 & 1424.7 & 1427.9 & 1431.0 & 1434. 2 & 1437.4 & 1440.6 & 4. 000 \\
\hline 4. 100 & 1440.6 & 1443.8 & 1446.9 & 1450.1 & 1453. 2 & 1456.4 & 1459.6 & 1462.7 & 1465.9 & 1469.0 & 1472.2 & 4. 100 \\
\hline $\begin{array}{l}4.2 \\
4.3\end{array}$ & $\begin{array}{l}1472.2 \\
1504.2\end{array}$ & $\begin{array}{l}1475.4 \\
1507.4\end{array}$ & $\begin{array}{l}1478.6 \\
1510.6\end{array}$ & $\begin{array}{l}1481.8 \\
1513.8\end{array}$ & $\begin{array}{l}1485.0 \\
15170\end{array}$ & 1488. 2 & 1491.4 & $\begin{array}{l}1494.6 \\
1526.5\end{array}$ & $\begin{array}{l}1497.8 \\
15297\end{array}$ & $\begin{array}{l}1501.0 \\
1532.9\end{array}$ & $\begin{array}{l}1504.2 \\
1536.1\end{array}$ & 4. 200 \\
\hline & & 15393 & 15425 & 1513.8 & 15480 & 15521 & 1523.3 & 15585 & 15617 & 15649 & & \\
\hline 4. 500 & $\begin{array}{l}1536.1 \\
1568.1\end{array}$ & $\begin{array}{l}1539.3 \\
1571.3\end{array}$ & $\begin{array}{l}1542.5 \\
1574.5\end{array}$ & $\begin{array}{l}1545.7 \\
1577.7\end{array}$ & $\begin{array}{l}1548.9 \\
1580.9\end{array}$ & $\begin{array}{l}1552.1 \\
1584.2\end{array}$ & $\begin{array}{l}1555.3 \\
1587.4\end{array}$ & $\begin{array}{l}1558.5 \\
1590.6\end{array}$ & $\begin{array}{l}1561.7 \\
1593.8\end{array}$ & $\begin{array}{l}1564.9 \\
1597.1\end{array}$ & $\begin{array}{l}1560.1 \\
1600.3\end{array}$ & $\begin{array}{l}4.400 \\
3.500\end{array}$ \\
\hline 4.600 & 1600.3 & 1603.6 & 1606.8 & 1610.1 & 1613. 3 & 1616.6 & 1619.9 & 1623.1 & 1626.4 & 1629.6 & 1632.9 & 4. 600 \\
\hline $\begin{array}{l}\text { 4. } 700 \\
4.800\end{array}$ & 1632.9 & 1636.1 & 1639.4 & 1642.6 & 1645.8 & 1649.1 & 1652.3 & 1655.5 & 1658. 7 & 1662.0 & 1665. 2 & 4. 700 \\
\hline $\begin{array}{l}4.800 \\
4.900\end{array}$ & $\begin{array}{l}1665.2 \\
1697.7\end{array}$ & $\begin{array}{l}1668.4 \\
1701.1\end{array}$ & $\begin{array}{l}1671.7 \\
1704.3\end{array}$ & $\begin{array}{l}1674.9 \\
1707.6\end{array}$ & $\begin{array}{l}1678.2 \\
1710.8\end{array}$ & $\begin{array}{l}1681.4 \\
1714.1\end{array}$ & $\begin{array}{l}1684.7 \\
1717.4\end{array}$ & $\begin{array}{l}1687.9 \\
1720.7\end{array}$ & $\begin{array}{l}1691.1 \\
1724.0\end{array}$ & & 1697.7 & $\begin{array}{r}4.800 \\
4.900\end{array}$ \\
\hline 5.000 & 1730.6 & 1733.9 & & & & & & & & & & \\
\hline & 1700.0 & 160.9 & 1737.1 & 1840.4 & 1845.7 & 1746.9 & 1750.2 & 1753.5 & 1756.8 & 1760.0 & 1763.3 & 5.000 \\
\hline $\begin{array}{l}5.100 \\
5.200\end{array}$ & $\begin{array}{l}1763.3 \\
1796.3\end{array}$ & $\begin{array}{l}1766.6 \\
1799.6\end{array}$ & 1769.9 & 1773. 2 & 1776.5 & 1779.8 & 1783.1 & 1786.4 & 1789. 7 & 1793.0 & 1796.3 & 5. 100 \\
\hline 5.300 & $\begin{array}{l}1796.3 \\
1829.3\end{array}$ & $\begin{array}{l}1799.6 \\
1832.6\end{array}$ & $\begin{array}{l}1802.9 \\
1836.0\end{array}$ & $\begin{array}{l}1806.2 \\
1839.3\end{array}$ & $\begin{array}{l}1809.5 \\
1842.6\end{array}$ & $\begin{array}{l}1812.8 \\
1845.9\end{array}$ & $\begin{array}{l}1816.1 \\
1849.3\end{array}$ & $\begin{array}{l}1819.4 \\
1852.6\end{array}$ & $\begin{array}{l}1822.7 \\
1855.9\end{array}$ & $\begin{array}{l}1826.0 \\
1859.3\end{array}$ & $\begin{array}{l}1829.3 \\
1862.6\end{array}$ & $\begin{array}{l}\text { 5. } 200 \\
5,300\end{array}$ \\
\hline 5. 400 & 1862.6 & 1865.9 & 1869.2 & & 1875 & 1879 & 882 & & & & & \\
\hline & 1895.7 & 0 & 1902. & 1905.6 & $\begin{array}{l}1875.9 \\
1908.9\end{array}$ & 1912 . & $\begin{array}{l}188 \\
191:\end{array}$ & $\begin{array}{l}1885.8 \\
1918.8\end{array}$ & $\begin{array}{l}1889.1 \\
192.1\end{array}$ & $\begin{array}{l}1892.4 \\
1925.4\end{array}$ & $\begin{array}{l}1895.7 \\
1928.7\end{array}$ & $\begin{array}{l}5.400 \\
5.500\end{array}$ \\
\hline 5. 600 & 1928.7 & 1932.0 & 1935.3 & 1938.7 & 1942.0 & 1945.3 & 1948.7 & $\begin{array}{l}1952.0 \\
1950\end{array}$ & $\begin{array}{l}1922.1 \\
1955.3\end{array}$ & $\begin{array}{l}1920.4 \\
1958.7\end{array}$ & 1962.0 & 5.600 \\
\hline 5. 700 & 1962. 0 & 1965.3 & 1968.7 & 1972.0 & 1975.3 & 1978.6 & 1982.0 & 1985.3 & 1988.6 & 1992.0 & 1995. 3 & 5. 700 \\
\hline $\begin{array}{l}5.800 \\
5.960\end{array}$ & $\begin{array}{l}1995.3 \\
2028.7\end{array}$ & $\begin{array}{l}1998.6 \\
2032.0\end{array}$ & 2035.4 & $\begin{array}{l}2005.3 \\
2038.7\end{array}$ & $\begin{array}{l}2008.7 \\
2042.0\end{array}$ & $\begin{array}{l}2012.0 \\
2045.3\end{array}$ & $\begin{array}{l}2015.3 \\
2048.7\end{array}$ & $\begin{array}{l}2018.7 \\
2052.0\end{array}$ & $\begin{array}{l}2022.0 \\
2055.3\end{array}$ & $\begin{array}{l}2025.4 \\
2058.7\end{array}$ & $\begin{array}{l}2028.7 \\
2062.0\end{array}$ & $\begin{array}{l}5.800 \\
5.900\end{array}$ \\
\hline 6.000 & 2062.0 & 20653 & 20686 & 20720 & 2075.3 & & 20820 & 20853 & 20886 & 0 & 2095.3 & 6.000 \\
\hline & & & & & & & & & & & & 6.000 \\
\hline $\begin{array}{l}6.100 \\
6.200\end{array}$ & $\begin{array}{l}2095.3 \\
2129.0\end{array}$ & $\begin{array}{l}2098.7 \\
2132\end{array}$ & $\begin{array}{l}2102.0 \\
2135.7\end{array}$ & $\begin{array}{l}2105.4 \\
2139.1\end{array}$ & $\begin{array}{l}2108.8 \\
2142.5\end{array}$ & $\begin{array}{l}2112.1 \\
2\end{array}$ & $\begin{array}{l}2115.5 \\
2149\end{array}$ & $\begin{array}{l}2118.9 \\
-1526\end{array}$ & $\begin{array}{l}2122.2 \\
2156.0\end{array}$ & $\begin{array}{l}2125.6 \\
2159.3\end{array}$ & $\begin{array}{l}2129.0 \\
2162.7\end{array}$ & 6. 100 \\
\hline $\begin{array}{l}0.200 \\
6.300\end{array}$ & $\begin{array}{l}2129.0 \\
2162.7\end{array}$ & $\begin{array}{l}2132.4 \\
2166.0\end{array}$ & $\begin{array}{l}2135.7 \\
2169.4\end{array}$ & $\begin{array}{l}2139.1 \\
2172.8\end{array}$ & $\begin{array}{l}2142.5 \\
2176.1\end{array}$ & $\begin{array}{l}2145.9 \\
2179.5\end{array}$ & $\begin{array}{l}2149.2 \\
2182.8\end{array}$ & $\begin{array}{l}2152.6 \\
2186.2\end{array}$ & $\begin{array}{l}2156.0 \\
2189.5\end{array}$ & $\begin{array}{l}2159.3 \\
2192.9\end{array}$ & $\begin{array}{l}2162 . \\
2196.2\end{array}$ & $\begin{array}{l}6.200 \\
6.300\end{array}$ \\
\hline 6. 400 & 2196.2 & 2199.5 & 2202.9 & 2206.2 & 2209.6 & 2212.9 & 2216.3 & 2219.6 & 2223.0 & 2226.3 & 2229.7 & 400 \\
\hline 6.500 & 2229.7 & 2233.1 & 2236.5 & 2239.2 & 2243.3 & 2246 . & 2250 & 2253.5 & 2256 . & 2260.3 & 226 & $\begin{array}{l}6.400 \\
6.500\end{array}$ \\
\hline 6.600 & 2263.7 & 2267.1 & 2270.4 & 2273.8 & 2277.2 & 2280.5 & 2283.9 & 2287.2 & 2290.6 & 2293.9 & 2297.3 & 6.600 \\
\hline 6.700 & 2297.3 & 2300.7 & 2304. 0 & 2307.4 & 2310.8 & 2314.1 & 2317.5 & 2320.9 & 2324.2 & 2327.6 & 2331.0 & 6. 700 \\
\hline $\begin{array}{l}6.800 \\
6.900\end{array}$ & $\begin{array}{l}2331.0 \\
2364.7\end{array}$ & $\begin{array}{l}2334.4 \\
2368.1\end{array}$ & $\begin{array}{l}2337.7 \\
2371.4\end{array}$ & $\begin{array}{l}2341.1 \\
2374.8\end{array}$ & $\begin{array}{l}2344.5 \\
2378.1\end{array}$ & $\begin{array}{l}2347.9 \\
2381.5\end{array}$ & $\begin{array}{l}2351.2 \\
2384.9\end{array}$ & $\begin{array}{l}2354.6 \\
2388.2\end{array}$ & $\begin{array}{l}2358.0 \\
2391.6\end{array}$ & $\begin{array}{l}2361.3 \\
2394.9\end{array}$ & $\begin{array}{l}2364.7 \\
2398.3\end{array}$ & $\begin{array}{l}6.800 \\
6.900\end{array}$ \\
\hline 7.000 & 2398.3 & 2401.7 & 2405.0 & 2408.4 & 2411.8 & 2415.2 & 2418.5 & 2421.9 & 2425.3 & 2428.6 & 2432.0 & 7.000 \\
\hline 7.100 & 2432.0 & 2435.3 & 2438.7 & 2442.0 & 2445.3 & 2448.7 & 2452.0 & 2455.3 & 2458.6 & 2462.0 & 2465.3 & 7.100 \\
\hline $\begin{array}{l}7.200 \\
7.300\end{array}$ & $\begin{array}{r}2465.3 \\
2498.7\end{array}$ & $\begin{array}{l}2468.6 \\
2502\end{array}$ & 2472.0 & 2475.3 & 2478 & 2482 & 2485.4 & 2488.7 & 249 & 2495.4 & & \\
\hline 7.300 & 2498.7 & 2502.0 & 2505.3 & 2508.6 & 2511.9 & 2515.2 & 2518.5 & 2521.8 & 2525.1 & 2528.4 & 2531.7 & 7.300 \\
\hline $\begin{array}{l}7.400 \\
7\end{array}$ & 2531.7 & 2535.0 & 2538. 3 & 2541.6 & 2544.9 & 2548.2 & 2551.5 & 2554.8 & 2558.1 & 2561.4 & 2564.7 & 7. 400 \\
\hline $\begin{array}{l}7.500 \\
7.600\end{array}$ & $\begin{array}{l}2564.7 \\
2597.7\end{array}$ & $\begin{array}{l}2568.0 \\
2601.0\end{array}$ & $\begin{array}{l}2571.3 \\
2604.2\end{array}$ & $\begin{array}{l}2574.6 \\
2607.5\end{array}$ & $\begin{array}{l}2577.9 \\
2610.8\end{array}$ & $\begin{array}{l}2581.2 \\
2614.0\end{array}$ & $\begin{array}{l}2584.5 \\
2617.3\end{array}$ & $\begin{array}{l}2587.8 \\
2620.5\end{array}$ & $\begin{array}{l}2591.1 \\
2623.8\end{array}$ & $\begin{array}{l}2594.4 \\
2627.0\end{array}$ & $\begin{array}{l}2597.7 \\
2630.3\end{array}$ & $\begin{array}{l}7.500 \\
7.600\end{array}$ \\
\hline 7.700 & 2630.3 & 2633 & 2636.8 & 2640 & 2643 & 264 & 264 & 265 & 265 & & & 7.700 \\
\hline 7. & 2662.9 & 266 & 266 & 2 & 0 & & & & & $\begin{array}{l}200 \\
269\end{array}$ & $\begin{array}{l}20095.9 \\
269.5\end{array}$ & $\because 800$ \\
\hline 7. 900 & 2695.5 & 2698.7 & 2702.0 & 2705.2 & 2708.4 & 2711.6 & 2714.9 & 2718.1 & 2721.3 & 2724.5 & 2727.7 & 7.900 \\
\hline 8.000 & 2727.7 & 2730.9 & 2734.1 & 2737.3 & 2740.5 & 2743.7 & 2746.9 & 2750.1 & 2753.3 & 2756.5 & 2759.7 & 8.000 \\
\hline 8.100 & 2759.7 & 2762.9 & 2766.0 & 2769.2 & 2772.3 & 2775 & 2778 & 2781.8 & 2784.9 & 2788.1 & 2791.2 & 8.100 \\
\hline & 2791.2 & 2794 & 2797.5 & & & & & & & & & \\
\hline 8.300 & 2822.5 & 2825.6 & 2828.8 & 2831.9 & 2835.0 & 2838.2 & 2841.3 & 2844.5 & 2847.6 & 2850.7 & 2853.9 & 8. 300 \\
\hline 8. 400 & 2853.9 & 2857.1 & 2860.2 & 2863.4 & 2866.6 & 2869.7 & 2872.9 & 2876.1 & 2879.3 & 28 & 6 & 8. 400 \\
\hline $\begin{array}{l}8.500 \\
8.600\end{array}$ & $\begin{array}{l}2885.6 \\
2917.1\end{array}$ & $\begin{array}{l}2888.7 \\
2920.3\end{array}$ & $\begin{array}{l}2891.9 \\
2923.5\end{array}$ & 2926.7 & & $\begin{array}{l}2901.3 \\
2933.0\end{array}$ & $\begin{array}{l}2904.5 \\
2936.2\end{array}$ & $\begin{array}{l}2907.6 \\
2939.4\end{array}$ & & & $\begin{array}{l}2917.1 \\
2949.0\end{array}$ & 500 \\
\hline & & & & 2926.7 & $292:$ & 29.00 .0 & & & 2942.0 & 2945.8 & & 8.600 \\
\hline $\begin{array}{l}8.700 \\
8.800\end{array}$ & $\begin{array}{l}2949.0 \\
2980.6\end{array}$ & $\begin{array}{l}2952.2 \\
2983.8\end{array}$ & $\begin{array}{l}2955.3 \\
2086.9\end{array}$ & 2958.5 & 2961.7 & $\begin{array}{l}2964.8 \\
\end{array}$ & 2968.0 & 2971.1 & 2974. 3 & 2977.4 & 2980.6 & 8. 700 \\
\hline $\begin{array}{l}8.800 \\
8.900\end{array}$ & $\begin{array}{l}2980.0 \\
3012.2\end{array}$ & $\begin{array}{l}2983.8 \\
3015.3\end{array}$ & $\begin{array}{l}2986.9 \\
3018.5\end{array}$ & & & 3027.8 & & & 3037 & 3040.3 & & $\begin{array}{l}8.800 \\
8.900\end{array}$ \\
\hline 9.000 & 3043.4 & 3046.5 & 3049.7 & 3052.8 & 3055.9 & 3059.1 & 3062.2 & 3065.3 & & 3071.6 & 3074.7 & 9.000 \\
\hline 9.100 & 3074 & 3077.8 & 30 & & & & & & 3099,4 & & & 0 \\
\hline & & & & & & & & & & & & $\begin{array}{l}9.100 \\
9.200\end{array}$ \\
\hline 9 & 3136 . & 3139 . & 3142.7 & 3145.8 & 3148.9 & 3151.9 & 3155.0 & 3158. & $\begin{array}{l}5100 . \\
3161 .\end{array}$ & 3164. 2 & $\begin{array}{l}\text { D10.0 } \\
3167.2\end{array}$ & 9. 300 \\
\hline & 3167.2 & 3170.2 & 3173.3 & 3176.3 & 3179.4 & 3182.4 & 3185 . & 3188 & 319 & 319 & 319 & 9. \\
\hline $\begin{array}{l}9.500 \\
9.500\end{array}$ & 3197.6 & & & & & & & & & & & \\
\hline 9.600 & 3227.9 & 3230. & 3234 . & 3237. & 3240 . & 3243. & 3246. & 3249 & 3252 . & 3255 & 3258.2 & 9.600 \\
\hline $\begin{array}{l}9.700 \\
9.800\end{array}$ & 3258.2 & 3261.2 & 3264.3 & 3267.3 & 3270.4 & 3273.4 & 3276.4 & 3279.4 & 3282.5 & 3285.5 & 3288.5 & 8. 700 \\
\hline $\begin{array}{l}9.800 \\
9.900\end{array}$ & $\begin{array}{l}3288.5 \\
3318.2\end{array}$ & $\begin{array}{l}3291.5 \\
3321.2\end{array}$ & $\begin{array}{l}3294.5 \\
3324.1\end{array}$ & $\begin{array}{l}3297.4 \\
3327.1\end{array}$ & $\begin{array}{l}3300.4 \\
3330.1\end{array}$ & $\begin{array}{l}3303.4 \\
3333.0\end{array}$ & $\begin{array}{l}3306.4 \\
3336.0\end{array}$ & $\begin{array}{l}3309.3 \\
3339.0\end{array}$ & $\begin{array}{l}3312.3 \\
3342.0\end{array}$ & $\begin{array}{l}3315.2 \\
3344.9\end{array}$ & $\begin{array}{l}3318.2 \\
3347.9\end{array}$ & $\begin{array}{l}9.800 \\
9.900\end{array}$ \\
\hline Millivolts & .000 & .010 & .020 & .030 & .040 & .050 & .060 & .070 & .080 & 090 & .100 & volts \\
\hline & & & & & & & & & & & & \\
\hline
\end{tabular}


TABLE III-A. Forty percent iridium-50 percent rhodium versus iridium thermocouples-Continued

Electromotive force in absolute millivolts. Temperature in degrees F (Int. 1948). Reference junction at $32{ }^{\circ} \mathrm{F}$.

\begin{tabular}{|c|c|c|c|c|c|c|c|c|c|c|c|c|}
\hline Millivolts & .000 & .010 & .020 & .030 & .040 & .050 & .060 & .070 & .080 & .090 & .100 & Millivolts \\
\hline 10.000 & 3347.9 & 3350.9 & 3353.8 & 3356.8 & 3359.8 & 3362.8 & 3365.7 & 3368.7 & 3371.7 & 3374.6 & 3377.6 & 10. 000 \\
\hline 10. 100 & 3377.6 & 3380.6 & 3383.5 & 3386.5 & 3389.4 & 3392.4 & 3395. 3 & 3398.3 & 3401.2 & 3404.2 & 3407.1 & 10. 100 \\
\hline $\begin{array}{l}10.200 \\
10.300\end{array}$ & $\begin{array}{l}3407.1 \\
3436.3\end{array}$ & $\begin{array}{l}3410.0 \\
3439.2\end{array}$ & $\begin{array}{l}3413.0 \\
3442.1\end{array}$ & $\begin{array}{l}3415.9 \\
3444.9\end{array}$ & $\begin{array}{l}3418.8 \\
3447.8\end{array}$ & $\begin{array}{l}3421.7 \\
3450.7\end{array}$ & $\begin{array}{l}3424.7 \\
3453.5\end{array}$ & 342 & $\begin{array}{l}3430.5 \\
3459.3\end{array}$ & $\begin{array}{l}3433.4 \\
346 .\end{array}$ & 3436.3 & 10. 200 \\
\hline 18 & 346 & 3467 & 3470 & 3473 & 34 & 34 & 348 & 348 & 0 & $300-8$ & & 00 \\
\hline 10. 500 & 3493. 7 & 3496. 6 & 3499.4 & $\begin{array}{l}5+10.0 \\
3502.3\end{array}$ & $\begin{array}{l}5+10.0 \\
3505.1\end{array}$ & $\begin{array}{l}077.4 .7 \\
3508.0\end{array}$ & 3510.8 & $\begin{array}{l}5460.1 \\
3513.7\end{array}$ & $\begin{array}{l}3488.0 \\
3516.5\end{array}$ & $\begin{array}{l}0780.0 \\
3519.4\end{array}$ & $\begin{array}{l}3495.1 \\
3522.2\end{array}$ & $\begin{array}{l}10.400 \\
10.500\end{array}$ \\
\hline 10.600 & 3522.2 & 3525.0 & 3527.8 & 3530.7 & 3533.5 & 3536.3 & 3539.1 & 3541.9 & 3544.7 & 3547.5 & 3550.3 & 10.600 \\
\hline 10. 700 & 3550.3 & 3553.1 & 3555.9 & 3558.7 & 3561.4 & 3564.2 & 3567. 0 & 3569.8 & 3572.6 & 3575 . & 3578.1 & 10. 700 \\
\hline $\begin{array}{l}10.800 \\
10.900\end{array}$ & $\begin{array}{l}3578.1 \\
3605.8\end{array}$ & $\begin{array}{l}3580.9 \\
3608.6\end{array}$ & $\begin{array}{l}3583.7 \\
3611.3\end{array}$ & $\begin{array}{l}3586.4 \\
3614.1\end{array}$ & $\begin{array}{l}3589.2 \\
3616.8\end{array}$ & $\begin{array}{l}3592.0 \\
3619.6\end{array}$ & $\begin{array}{l}3594.7 \\
3622.3\end{array}$ & $\begin{array}{l}3597.5 \\
3625.1\end{array}$ & $\begin{array}{l}3600.3 \\
3627.8\end{array}$ & $\begin{array}{l}3603.0 \\
3630.6\end{array}$ & $\begin{array}{l}3605.8 \\
3633.3\end{array}$ & $\begin{array}{l}10.800 \\
10.900\end{array}$ \\
\hline 11.000 & 3633.3 & 3636.0 & 3638.8 & 3641.5 & 3644.3 & 3647.0 & 3649.8 & 3652.5 & 3655.3 & 3658.0 & 3660.8 & 11.000 \\
\hline 11.100 & 3660.8 & 3663.6 & 3666.3 & 3669.1 & 3671.8 & 3674.6 & 3677.3 & 3680.1 & 3682.8 & 3685.6 & 3688.3 & 11. 100 \\
\hline $\begin{array}{l}11.200 \\
11.300\end{array}$ & $\begin{array}{l}3688.3 \\
3715.4\end{array}$ & $\begin{array}{l}3691.0 \\
3718.2\end{array}$ & $\begin{array}{l}3693.7 \\
3720.9\end{array}$ & $\begin{array}{l}3696.4 \\
3723.7\end{array}$ & $\begin{array}{l}3699.1 \\
3726.4\end{array}$ & $\begin{array}{l}3701.8 \\
3729.2\end{array}$ & $\begin{array}{l}3704.5 \\
3732.0\end{array}$ & $\begin{array}{l}3707.3 \\
3734.7\end{array}$ & $\begin{array}{l}3710.0 \\
3737.5\end{array}$ & $\begin{array}{l}3712.7 \\
3740.2\end{array}$ & $\begin{array}{l}3715.4 \\
3743.0\end{array}$ & $\begin{array}{l}\text { 11. } 200 \\
11.300\end{array}$ \\
\hline 11. 400 & 3743.0 & 3745.7 & 3748.5 & 3751.2 & 3754.0 & 375 & 3759.4 & 3762.1 & 3764.9 & $37 t$ & 3770.3 & 11.400 \\
\hline 500 & 3770.3 & 3773.0 & $\begin{array}{l}3775.7 \\
3775\end{array}$ & 3778.4 & 3781.1 & 3783.8 & $\begin{array}{l}3786.5 \\
378.5\end{array}$ & $\begin{array}{l}3789.2 \\
378.2\end{array}$ & $\begin{array}{l}3791.9 \\
370.9\end{array}$ & $\begin{array}{l}3794.6 \\
3796\end{array}$ & 3797. 3 & 11. 500 \\
\hline & & & & & & & & & & & & \\
\hline Millivolts & .000 & .010 & .020 & .030 & .040 & .050 & .060 & .070 & .080 & .090 & .100 & Millivolts \\
\hline
\end{tabular}

TABLE IV-A. Forty percent iridium-60 percent rhodium versus iridium thermocouples

Electromotive force in absolute millivolts. Temperature in degrees $\mathrm{F}$ (Int. 1948). Reference junction at $32{ }^{\circ} \mathrm{F}$.

\begin{tabular}{|c|c|c|c|c|c|c|c|c|c|c|c|c|}
\hline${ }^{\circ} \mathrm{F}$ & 0 & 10 & 20 & 30 & 40 & 50 & 60 & 70 & 80 & 90 & 100 & ${ }^{\circ} \mathrm{F}$ \\
\hline $\begin{array}{c}0 \\
100 \\
200 \\
300 \\
400\end{array}$ & $\begin{array}{r}0.126 \\
.337 \\
.576 \\
.840\end{array}$ & $\begin{array}{r}0.146 \\
.360 \\
.602 \\
.868\end{array}$ & $\begin{array}{r}0.166 \\
.383 \\
.627 \\
.895\end{array}$ & $\begin{array}{r}0.186 \\
.406 \\
.653 \\
.923\end{array}$ & $\begin{array}{r}0.015 \\
.206 \\
.430 \\
.679 \\
.951\end{array}$ & $\begin{array}{r}0.032 \\
.277 \\
.454 \\
.706 \\
.979\end{array}$ & $\begin{array}{r}0.050 \\
.249 \\
.478 \\
.732 \\
1.007\end{array}$ & $\begin{array}{r}0.068 \\
.271 \\
.502 \\
.759 \\
1.036\end{array}$ & $\begin{array}{r}0.087 \\
.293 \\
.526 \\
.786 \\
1.064\end{array}$ & $\begin{array}{r}0.106 \\
.315 \\
.551 \\
.813 \\
1.093\end{array}$ & $\begin{array}{r}0.126 \\
.337 \\
.576 \\
.840 \\
1.122\end{array}$ & $\begin{array}{c}0 \\
100 \\
200 \\
300 \\
400\end{array}$ \\
\hline $\begin{array}{l}500 \\
600 \\
700 \\
800 \\
900\end{array}$ & $\begin{array}{l}\text { 1. } 122 \\
\text { 1. } 418 \\
\text { 1. } 726 \\
\text { 2. } 041 \\
\text { 2. } 361\end{array}$ & $\begin{array}{l}1.151 \\
1.449 \\
1.757 \\
2.073 \\
2.393\end{array}$ & $\begin{array}{l}1.180 \\
1.480 \\
1.788 \\
2.105 \\
2.425\end{array}$ & $\begin{array}{l}1.209 \\
1.510 \\
1.820 \\
2.137 \\
2.458\end{array}$ & $\begin{array}{l}\text { 1. } 239 \\
\text { 1. } 541 \\
\text { 1. } 851 \\
\text { 2. } 169 \\
\text { 2. } 490\end{array}$ & $\begin{array}{l}\text { 1. } 268 \\
1.571 \\
1.883 \\
2.201 \\
2.522\end{array}$ & $\begin{array}{l}1.298 \\
1.602 \\
1.915 \\
2.233 \\
2.554\end{array}$ & $\begin{array}{l}1.328 \\
1.633 \\
\text { 1. } 946 \\
2.265 \\
2.587\end{array}$ & $\begin{array}{l}\text { 1. } 358 \\
\text { 1. } 664 \\
\text { 1. } 978 \\
\text { 2. } 297 \\
\text { 2. } 619\end{array}$ & $\begin{array}{l}1.388 \\
1.695 \\
2.010 \\
2.329 \\
2.652\end{array}$ & $\begin{array}{l}1.418 \\
\text { 1. } 726 \\
\text { 2. } 041 \\
2.361 \\
2.684\end{array}$ & $\begin{array}{l}500 \\
600 \\
700 \\
800 \\
900\end{array}$ \\
\hline $\begin{array}{l}1000 \\
1100 \\
1200 \\
1300 \\
1400\end{array}$ & $\begin{array}{l}\text { 2. } 684 \\
\text { 3. } 009 \\
\text { 3. } 332 \\
\text { 3. } 654 \\
\text { 3. } 973\end{array}$ & $\begin{array}{l}\text { 2. } 717 \\
\text { 3. } 041 \\
\text { 3. } 365 \\
\text { 3. } 686 \\
\text { 4. } 004\end{array}$ & $\begin{array}{l}2.749 \\
3.073 \\
3.397 \\
3.718 \\
4.036\end{array}$ & $\begin{array}{l}2.781 \\
3.106 \\
3.429 \\
3.750 \\
4.067\end{array}$ & $\begin{array}{l}\text { 2. } 814 \\
\text { 3. } 138 \\
\text { 3. } 461 \\
\text { 3. } 782 \\
\text { 4. } 098\end{array}$ & $\begin{array}{l}2.846 \\
3.170 \\
3.494 \\
3.814 \\
4.130\end{array}$ & $\begin{array}{l}\text { 2. } 878 \\
\text { 3. } 203 \\
\text { 3. } 526 \\
\text { 3. } 846 \\
\text { 4. } 162\end{array}$ & $\begin{array}{l}\text { 2. } 911 \\
\text { 3. } 235 \\
\text { 3. } 558 \\
\text { 3. } 878 \\
\text { 4. } 193\end{array}$ & $\begin{array}{l}2.943 \\
3.267 \\
3.590 \\
3.910 \\
4.225\end{array}$ & $\begin{array}{l}2.976 \\
\text { 3. } 299 \\
\text { 3. } 622 \\
\text { 3. } 941 \\
\text { 4. } 256\end{array}$ & $\begin{array}{l}\text { 3. } 009 \\
\text { 3. } 332 \\
\text { 3. } 654 \\
\text { 3. } 973 \\
\text { 4. } 287\end{array}$ & $\begin{array}{l}1000 \\
1100 \\
1200 \\
1300 \\
1400\end{array}$ \\
\hline $\begin{array}{l}1500 \\
1600 \\
1700 \\
1800 \\
1900\end{array}$ & $\begin{array}{l}\text { 4. } 287 \\
\text { 4. } 599 \\
\text { 4. } 907 \\
\text { 5. } 211 \\
\text { 5. } 513\end{array}$ & $\begin{array}{l}\text { 4. } 318 \\
\text { 4. } 629 \\
\text { 4. } 937 \\
\text { 5. } 242 \\
\text { 5. } 543\end{array}$ & $\begin{array}{l}\text { 4. } 349 \\
\text { 4. } 660 \\
\text { 4. } 968 \\
\text { 5. } 272 \\
\text { 5. } 574\end{array}$ & $\begin{array}{l}4.381 \\
4.691 \\
4.998 \\
5.302 \\
5.604\end{array}$ & $\begin{array}{l}\text { 4. } 412 \\
\text { 4. } 722 \\
\text { 5. } 029 \\
\text { 5. } 332 \\
\text { 5. } 634\end{array}$ & $\begin{array}{l}4.443 \\
4.753 \\
5.060 \\
5.362 \\
5.664\end{array}$ & $\begin{array}{l}\text { 4. } 474 \\
4.784 \\
5.090 \\
5.392 \\
5.694\end{array}$ & $\begin{array}{l}4.506 \\
\text { 4. } 815 \\
5.120 \\
5.423 \\
5.724\end{array}$ & $\begin{array}{l}4.537 \\
4.846 \\
5.150 \\
5.453 \\
5.754\end{array}$ & $\begin{array}{l}\text { 4. } 568 \\
4.876 \\
5.181 \\
5.483 \\
5.784\end{array}$ & $\begin{array}{l}\text { 4. } 599 \\
\text { 4. } 907 \\
5.211 \\
5.513 \\
5.814\end{array}$ & $\begin{array}{l}1500 \\
1600 \\
1700 \\
1800 \\
1900\end{array}$ \\
\hline $\begin{array}{l}2000 \\
2100 \\
2200 \\
2300 \\
2400\end{array}$ & $\begin{array}{l}\text { 5. } 814 \\
\text { 6. } 114 \\
6.411 \\
6.708 \\
7.005\end{array}$ & $\begin{array}{l}5.844 \\
6.143 \\
6.441 \\
6.738 \\
7.035\end{array}$ & $\begin{array}{l}5.874 \\
6.173 \\
6.471 \\
6.767 \\
7.064\end{array}$ & $\begin{array}{l}5.904 \\
6.203 \\
6.501 \\
6.797 \\
7.094\end{array}$ & $\begin{array}{l}\text { 5. } 934 \\
6.233 \\
6.530 \\
6.827 \\
7.124\end{array}$ & $\begin{array}{l}5.964 \\
6.263 \\
6.560 \\
6.857 \\
7.154\end{array}$ & $\begin{array}{l}5.994 \\
6.292 \\
6.589 \\
6.886 \\
7.184\end{array}$ & $\begin{array}{l}6.024 \\
6.322 \\
6.619 \\
6.916 \\
7.214\end{array}$ & $\begin{array}{l}6.054 \\
6.352 \\
6.648 \\
6.946 \\
7.244\end{array}$ & $\begin{array}{l}\text { 6. U84 } \\
\text { 6. } 382 \\
6.678 \\
6.976 \\
7.274\end{array}$ & $\begin{array}{l}\text { 6. } 114 \\
\text { 6. } 411 \\
6.708 \\
7.005 \\
7.304\end{array}$ & $\begin{array}{l}2000 \\
2100 \\
2200 \\
2300 \\
2400\end{array}$ \\
\hline $\begin{array}{l}2500 \\
2600 \\
2700 \\
2800 \\
2900\end{array}$ & $\begin{array}{l}7.304 \\
7.607 \\
7.914 \\
8.228 \\
8.545\end{array}$ & $\begin{array}{l}7.334 \\
7.638 \\
7.945 \\
8.260 \\
8.577\end{array}$ & $\begin{array}{l}7.364 \\
7.669 \\
7.976 \\
8.292 \\
8.608\end{array}$ & $\begin{array}{l}7.395 \\
7.699 \\
8.007 \\
8.324 \\
8.640\end{array}$ & $\begin{array}{l}7.425 \\
7.730 \\
8.039 \\
8.356 \\
8.672\end{array}$ & $\begin{array}{l}7.455 \\
7.760 \\
8.070 \\
8.388 \\
8.703\end{array}$ & $\begin{array}{l}7.486 \\
7.791 \\
8.101 \\
8.419 \\
8.735\end{array}$ & $\begin{array}{l}7.516 \\
7.822 \\
8.133 \\
8.451 \\
8.767\end{array}$ & $\begin{array}{l}7.547 \\
7.853 \\
8.164 \\
8.482 \\
8.798\end{array}$ & $\begin{array}{l}7.577 \\
7.883 \\
8.196 \\
8.514 \\
8.830\end{array}$ & $\begin{array}{l}\text { 7. } 607 \\
7.914 \\
\text { 8. } 228 \\
8.545 \\
8.862\end{array}$ & $\begin{array}{l}2500 \\
2600 \\
2780 \\
2800 \\
2900\end{array}$ \\
\hline $\begin{array}{l}3000 \\
3100 \\
3200 \\
3300 \\
3400\end{array}$ & $\begin{array}{r}8.862 \\
9.182 \\
9.508 \\
9.839 \\
10.176\end{array}$ & $\begin{array}{r}8.893 \\
9.214 \\
9.541 \\
9.873 \\
10.210\end{array}$ & $\begin{array}{r}8.925 \\
9.246 \\
9.574 \\
9.906 \\
10.244\end{array}$ & $\begin{array}{r}8.957 \\
9.279 \\
9.607 \\
9.940 \\
10.278\end{array}$ & $\begin{array}{r}8.989 \\
9.311 \\
9.640 \\
9.973 \\
10.313\end{array}$ & $\begin{array}{r}9.021 \\
9.344 \\
9.673 \\
10.007 \\
10.348\end{array}$ & $\begin{array}{r}9.053 \\
9.377 \\
9.706 \\
10.041 \\
10.383\end{array}$ & $\begin{array}{r}9.085 \\
9.409 \\
9.739 \\
10.075 \\
10.417\end{array}$ & $\begin{array}{r}9.117 \\
9.442 \\
9.772 \\
10.108 \\
10.452\end{array}$ & $\begin{array}{r}9.149 \\
9.475 \\
9.805 \\
10.142 \\
10.487\end{array}$ & $\begin{array}{r}9.182 \\
9.508 \\
9.839 \\
10.176 \\
10.522\end{array}$ & $\begin{array}{l}3000 \\
3100 \\
3200 \\
3300 \\
3400\end{array}$ \\
\hline $\begin{array}{l}3500 \\
3600 \\
3700 \\
3800\end{array}$ & $\begin{array}{l}10.522 \\
10.879 \\
11.243 \\
11.610\end{array}$ & $\begin{array}{l}10.557 \\
10.915 \\
11.280\end{array}$ & $\begin{array}{l}\text { 10. } 592 \\
10.951 \\
11.317\end{array}$ & $\begin{array}{l}10.628 \\
10.988 \\
11.353\end{array}$ & $\begin{array}{l}10.663 \\
11.024 \\
11.389\end{array}$ & $\begin{array}{l}10.699 \\
11.061 \\
11.426\end{array}$ & $\begin{array}{l}10.735 \\
11.097 \\
11.462\end{array}$ & $\begin{array}{l}\text { 10. } 771 \\
11.133 \\
11.499\end{array}$ & $\begin{array}{l}10.807 \\
11.170 \\
11.536\end{array}$ & $\begin{array}{l}10.843 \\
11.206 \\
11.573\end{array}$ & $\begin{array}{l}10.879 \\
11.243 \\
11.610\end{array}$ & $\begin{array}{l}3500 \\
3600 \\
3700 \\
3800\end{array}$ \\
\hline${ }^{\circ} \mathrm{F}$ & 0 & 10 & 20 & 30 & 40 & 50 & 60 & 70 & 80 & 90 & 100 & ${ }^{\circ} \mathrm{F}$ \\
\hline
\end{tabular}


TABLE V-A. Thermal emf of iridium versus platinum 27 Reference junction at $32^{\circ} \mathrm{F}$.

\begin{tabular}{|c|c|c|c|}
\hline${ }^{\circ} \mathrm{F}$ & $m v$ & ${ }^{\circ} \mathrm{F}$ & $m v$ \\
\hline $\begin{array}{r}32 \\
100 \\
150 \\
200 \\
250\end{array}$ & $\begin{array}{c}0 \\
0.227 \\
.413 \\
.616 \\
.834\end{array}$ & $\begin{array}{l}1300 \\
1350 \\
1400 \\
1450 \\
1500\end{array}$ & $\begin{array}{l}\text { 7. } 729 \\
8.158 \\
8.596 \\
9.043 \\
9.499\end{array}$ \\
\hline $\begin{array}{l}300 \\
350 \\
400 \\
450 \\
500\end{array}$ & $\begin{array}{l}1.066 \\
1.310 \\
1.565 \\
1.830 \\
2.105\end{array}$ & $\begin{array}{l}1550 \\
1600 \\
1650 \\
1700 \\
1750\end{array}$ & $\begin{array}{r}9.964 \\
10.437 \\
10.919 \\
11.410 \\
11.910\end{array}$ \\
\hline $\begin{array}{l}550 \\
600 \\
650 \\
700 \\
750\end{array}$ & $\begin{array}{l}2.390 \\
2.685 \\
2.988 \\
3.299 \\
3.619\end{array}$ & $\begin{array}{l}1800 \\
1850 \\
1900 \\
1950 \\
2000\end{array}$ & $\begin{array}{l}12.419 \\
12.936 \\
13.460 \\
13.992 \\
14.532\end{array}$ \\
\hline $\begin{array}{r}800 \\
850 \\
900 \\
950 \\
1000\end{array}$ & $\begin{array}{l}3.947 \\
4.284 \\
4.630 \\
4.985 \\
5.349\end{array}$ & $\begin{array}{l}2050 \\
2100 \\
2150 \\
2200 \\
2250\end{array}$ & $\begin{array}{l}15.079 \\
15.632 \\
16.190 \\
16.753 \\
17.321\end{array}$ \\
\hline $\begin{array}{l}1050 \\
1100 \\
1150 \\
1200\end{array}$ & $\begin{array}{l}5.723 \\
6.106 \\
6.498 \\
6.899\end{array}$ & $\begin{array}{l}2300 \\
2350 \\
2400 \\
2450\end{array}$ & $\begin{array}{l}17.893 \\
18.469 \\
19.048 \\
19.629\end{array}$ \\
\hline 1250 & 7. 309 & 2500 & 20. 211 \\
\hline
\end{tabular}

TABLE VI-A. Thermal emf of 40 percent iridum-60 percent rhodium versus copper and copper versus iridium thermocouples

\begin{tabular}{|c|c|c|}
\hline${ }^{\circ} \mathrm{F}$ & $\begin{array}{c}40 \% \text { Ir }-60 \% \\
\text { Rh vs Cu }\end{array}$ & Cu vs Ir \\
\hline $\begin{array}{r}32 \\
50 \\
75 \\
100 \\
125\end{array}$ & $\begin{array}{c}m v \\
0.000 \\
.026 \\
.064 \\
.103 \\
.141\end{array}$ & $\begin{array}{c}m v \\
0.000 \\
.006 \\
.014 \\
.023 \\
.035\end{array}$ \\
\hline $\begin{array}{l}150 \\
175 \\
200 \\
225 \\
250\end{array}$ & $\begin{array}{r}.178 \\
.217 \\
.253 \\
.289 \\
.325\end{array}$ & $\begin{array}{l}.049 \\
.065 \\
.084 \\
.105 \\
.129\end{array}$ \\
\hline $\begin{array}{l}275 \\
300 \\
350 \\
400 \\
450\end{array}$ & $\begin{array}{r}.359 \\
.392 \\
.459 \\
.525 \\
.584\end{array}$ & $\begin{array}{l}.155 \\
.184 \\
.247 \\
.315 \\
.395\end{array}$ \\
\hline 500 & .637 & .485 \\
\hline
\end{tabular}

(Paper 66C1-81) 